\title{
Estimating the Time Course of the Excitatory Synaptic Conductance in Neocortical Pyramidal Cells Using a Novel Voltage Jump Method
}

\author{
Michael Häusser ${ }^{1}$ and Arnd Roth ${ }^{2}$ \\ ${ }^{1}$ Laboratoire de Neurobiologie, Ecole Normale Supérieure, 75005 Paris, France, and ${ }^{2}$ Abteilung Zellphysiologie, \\ Max-Planck-Institut für Medizinische Forschung, 69120 Heidelberg, Germany
}

We introduce a method that permits faithful extraction of the decay time course of the synaptic conductance independent of dendritic geometry and the electrotonic location of the synapse. The method is based on the experimental procedure of Pearce (1993), consisting of a series of identical somatic voltage jumps repeated at various times relative to the onset of the synaptic conductance. The progression of synaptic charge recovered by successive jumps has a characteristic shape, which can be described by an analytical function consisting of sums of exponentials. The voltage jump method was tested with simulations using simple equivalent cylinder cable models as well as detailed compartmental models of pyramidal cells. The decay time course of the synaptic conductance could be estimated with high accuracy, even with high series resistances, low membrane resistances, and electrotonically remote, distrib- uted synapses. The method also provides the time course of the voltage change at the synapse in response to a somatic voltage-clamp step and thus may be useful for constraining compartmental models and estimating the relative electrotonic distance of synapses. In conjunction with an estimate of the attenuation of synaptic charge, the method also permits recovery of the amplitude of the synaptic conductance. We use the method experimentally to determine the decay time course of excitatory synaptic conductances in neocortical pyramidal cells. The relatively rapid decay time constant we have estimated $\left(\tau \sim 1.7 \mathrm{msec}\right.$ at $35^{\circ} \mathrm{C}$ ) has important consequences for dendritic integration of synaptic input by these neurons.

Key words: neocortex; pyramidal cell; space clamp; voltage clamp; cable modeling; synaptic current; EPSC
Knowledge of the time course of the synaptic conductance is of fundamental importance to our understanding of synaptic transmission. The kinetics of the synaptic conductance influences neuronal function in many ways, from shaping the resulting synaptic potential and setting the time window for synaptic integration to determining the synaptic charge (particularly relevant when a significant fraction of the current is carried by ions such as $\left.\mathrm{Ca}^{2+}\right)$. Furthermore, comparing synaptic conductance time course with receptor channel kinetics provides valuable information about the processes underlying synaptic transmission.

Synaptic conductance is conventionally measured by recording the synaptic current with somatic voltage clamp. In cells where all synapses are electrotonically close to or at the soma, such as cerebellar granule cells (Silver et al., 1992, 1995), neuroendocrine cells (Schneggenburger and Konnerth, 1992; Borst et al., 1994), unipolar brush cells (Rossi et al., 1995) and neurons in the auditory pathway (Forsythe and Barnes-Davies, 1993; Zhang and Trussell, 1994; Isaacson and Walmsley, 1995), this method can

\footnotetext{
Received May 27, 1997; revised July 24, 1997; accepted July 28, 1997.

This work was supported by the Centre National de la Recherche Scientifique, the Max-Planck-Gesellschaft, and a fellowship from the HFSP to M.H. Programs written for IGOR (Wavemetrics, Lake Oswego, OR) and Mathematica (Wolfram Research, Champaign, IL) incorporating various versions of the analytical function as fit routines are available on request. We thank Philippe Ascher and Bert Sakmann for their support, Nelson Spruston for carrying out some preliminary simulations, and Beverley Clark for help with experiments. We are also grateful to Boris Barbour, Guy Major, Nelson Spruston, and Greg Stuart for many useful discussions and Gerard Borst, Dirk Feldmeyer, Zach Mainen, Guy Major, and Angus Silver for their comments on this manuscript.

Correspondence should be addressed to Michael Häusser, Department of Physiology, University College London, Gower Street, London WC1E 6BT, UK.

Copyright (C) 1997 Society for Neuroscience $0270-6474 / 97 / 177606-20 \$ 05.00 / 0$
}

reliably measure the conductance time course. Alternatively, one can select for somatic synapses using cable model predictions (Finkel and Redman, 1983; Nelson et al., 1986). However, in most neurons, the majority of synapses are located at a considerable electrotonic distance from the soma, and therefore somatic voltage clamp of these synapses is associated with substantial attenuation and distortion of the synaptic current (Johnston and Brown, 1983; Rall and Segev, 1985; Major, 1993; Spruston et al., 1993; Mainen et al., 1996). This problem has proved to be rather intractable, and although several solutions have been proposed to date (see Discussion), none are completely satisfactory.

Recently an experimental technique was introduced by Pearce (1993), which uses somatic voltage jumps at various times during the synaptic conductance to determine how long after the onset of the synaptic current the synaptic conductance remains active. The principle of the technique is that a voltage jump that increases the synaptic driving force will only recover additional synaptic charge if the jump occurs while the conductance is still active. The technique was used to show that the GABAergic synaptic conductance generated by activation of distal synapses in hippocampal CA1 pyramidal neurons has a prominent slow component; however, a quantitative determination of the conductance time course was not made. This technique was subsequently applied to excitatory synapses in various neuronal types, also to demonstrate that the synaptic conductance at these synapses has a prolonged component (Barbour et al., 1994; Mennerick and Zorumski, 1995; Rossi et al., 1995; Kirson and Yaari, 1996).

Here we show using simulations in a variety of neuronal models that by measuring the time course of recovered charge this experimental technique can be used to determine the decay time course 
of the synaptic conductance with a high degree of accuracy. A simple analytical function providing a quantitative description of the results is presented, and limitations and potential applications of the method are explored. We use the method to estimate the time course of the excitatory synaptic conductance in neocortical pyramidal cells.

\section{MATERIALS AND METHODS}

\section{Simulations}

All simulations were performed using NEURON (Hines, 1993) running on Sun Sparcstations (Sun Microsystems, Mountain View, CA). The integration time step was $10 \mu \mathrm{sec}$. The synaptic conductance consisted of a sum of two or three exponentials, one for the rise (always $0.2 \mathrm{msec}$, unless otherwise indicated) and one or two for the decay. A "delta pulse" synaptic conductance was simulated using a $1 \mathrm{nS}$ conductance with duration of $0.1 \mathrm{msec}$. Except for the equivalent cylinder simulations and the simulations shown in Figure 8, synaptic contacts were placed at the head of explicitly modeled spines. The series resistance of the recording pipette was always $0.5 \mathrm{M} \Omega$, except where otherwise indicated, which is achievable in experiments using the neuronal types shown here (5 $\mathrm{M} \Omega$ compensated by $90 \%$ ). Unless otherwise indicated, the decay time constant of synaptic currents recorded at the soma was fit using a single exponential function, starting at the time point when the current had decayed to $\sim 90 \%$ of the peak amplitude.

Equivalent cylinder model. The geometry used in the equivalent cylinder simulation was as follows (see Fig. 1A): soma, $10 \mu \mathrm{m}$ long, $10 \mu \mathrm{m}$ diameter, 10 segments; and dendrite, $500 \mu \mathrm{m}$ long, $1.2 \mu \mathrm{m}$ diameter, 100 segments. Electrical parameters were: $R_{\mathrm{i}}=150 \Omega \mathrm{cm} ; R_{\mathrm{m}}=50,000 \Omega \mathrm{cm}^{2}$; and $C_{\mathrm{m}}=1.0 \mu \mathrm{F} \mathrm{cm}^{-2}$, giving an electrotonic length of the dendrite of $L=0.5$. The passive reversal potential was $-65 \mathrm{mV}$.

CA3 pyramidal cell model. The CA3 pyramidal cell model was based on cell CA3 15 in the article by Major et al. (1994), which is from a 19-d-old rat. The morphology was converted from the native format to that of NEURON using a program written in Mathematica (Wolfram Research, Champaign, IL). The electrotonic length of each segment was $<0.01$. The electrical parameters were $R_{\mathrm{i}}=250 \Omega \mathrm{cm} ; R_{\mathrm{m}}=180,000 \Omega \mathrm{cm}^{2}$; and $C_{\mathrm{m}}=0.66 \mu \mathrm{F} \mathrm{cm}^{-2}$; with a passive reversal potential of $-65 \mathrm{mV}$. Spine corrections were performed as described by Major et al. (1994), and the axon was not included in the simulations. The spine at the excitatory synaptic contact had a neck length of $0.66 \mu \mathrm{m}$, a neck diameter of $0.2 \mu \mathrm{m}$, a head length of $0.5 \mu \mathrm{m}$, and a head diameter of $0.45 \mu \mathrm{m}$.

Neocortical pyramidal cell model. The morphology of the layer 5 pyramidal cell was taken from the work of Markram et al. (1997) and comes from a postnatal day 14 rat (same neuron as shown in red in Markram et al., their Fig. 13). The electrotonic length of each segment was $<0.02$. The values for passive cable properties were $R_{\mathrm{i}}=150 \Omega \mathrm{cm} ; R_{\mathrm{m}}=30000$ $\Omega \mathrm{cm}^{2}$; and $C_{\mathrm{m}}=0.75 \mu \mathrm{F} \mathrm{cm}{ }^{-2}$, and the passive reversal potential was set to $-70 \mathrm{mV}$ (Mainen and Sejnowski, 1996). The measured dendritic membrane area was multiplied by a factor of 2 to account for spines. The axon was included, but axon collaterals were omitted. The neck length of the explicitly modeled spines was $1.0 \mu \mathrm{m}$, neck diameter was $0.35 \mu \mathrm{m}$, and head length and diameter were both $0.7 \mu \mathrm{m}$ (Peters and KaisermanAbramof, 1970).

Active conductances were added to the model as described in Mainen and Sejnowski (1996), based on the parameters in their original NEURON files (available via World Wide Web at http://www.cnl salk.edu/CNL/simulations.html). Two changes were made with respect to the original files of Mainen and Sejnowski (1996): (1) the reversal potential for $\mathrm{Ca}^{2+}$ was not constant at $+140 \mathrm{mV}$ but updated according to the Nernst equation assuming $\left[\mathrm{Ca}^{2+}\right]_{\mathrm{o}}=2 \mathrm{~mm}$; and 2) the time step was $10 \mu \mathrm{sec}$ instead of $25 \mu \mathrm{sec}$.

\section{Experiments}

Whole-cell patch-clamp recordings were made from the soma of visually identified thick tufted layer 5 pyramidal cells in slices of rat neocortex as described previously (Stuart et al., 1993; Markram et al., 1997). Wistar rats (14-18 d) were killed by decapitation, and sagittal neocortical slices $(250-300 \mu \mathrm{m})$ were cut on a Vibratome (Dosaka) in ice-cold extracellular solution containing (in $\mathrm{mM}$ ): $125 \mathrm{NaCl}, 2.5 \mathrm{KCl}, 25$ glucose, 25 $\mathrm{NaHCO}_{3}, 1.25 \mathrm{NaH}_{2} \mathrm{PO}_{4}, 2 \mathrm{CaCl}_{2}$, and $1 \mathrm{MgCl}_{2}$. The slices were incubated at $34^{\circ} \mathrm{C}$ for $45 \mathrm{~min}$ and then kept at room temperature before transfer to the recording chamber. With the use of an upright microscope (Axioskop, 40×-W/0.75 numerical aperture water-immersion objective;
Zeiss, Oberkochen, Germany) and infrared differential interference contrast videomicroscopy (Stuart et al., 1993), layer 5 pyramidal neurons were easily identified by their large somata, prominent axon initial segment, and thick apical dendrites projecting to higher layers.

Recordings were made using an Axopatch 200A amplifier (Axon Instruments, Foster City, CA). The internal patch pipette solution contained (in $\mathrm{mM}$ ): 100 potassium gluconate, $20 \mathrm{KCl}, 10 \mathrm{HEPES}, 10 \mathrm{EGTA}$, $4 \mathrm{Na}_{2}$-ATP, and $4 \mathrm{MgCl}_{2}$ (295 mOsm, $\mathrm{pH}$ adjusted to 7.3 with $\mathrm{KOH}$ ); in most experiments internal solutions also included $1 \mathrm{~mm}$ QX-314 (Alomone Laboratories) to block voltage-gated channels (particularly sodium channels) (Strichartz, 1973) and $0.5 \mathrm{~mm} \mathrm{ZD} 7288$ (Tocris) to block the hyperpolarization-activated cation current (Harris and Constanti, 1995). NMDA and $\mathrm{GABA}_{\mathrm{A}}$ receptors were blocked using $30 \mu \mathrm{M}$ D-APV, $50 \mu \mathrm{M}$ picrotoxin, and $50 \mu \mathrm{M}$ bicuculline methiodide, and $\mathrm{CaCl}_{2}$ and $\mathrm{MgCl}_{2}$ were increased to $3 \mathrm{~mm}$ to reduce polysynaptic activity. Membrane potentials were not corrected for the liquid junction potential. Currents were filtered at a bandwidth of $2 \mathrm{kHz}(-3 \mathrm{~dB})$ using an eight-pole low-pass Bessel filter and sampled at $20 \mathrm{kHz}$ using pCLAMP software (Axon Instruments). Series resistance (3-20 M $\Omega$; overall mean, $9.8 \pm 1.2 \mathrm{M} \Omega$ ) was monitored continuously and compensated by $85-90 \%$. All experiments were performed at $35 \pm 1^{\circ} \mathrm{C}$.

Excitatory synaptic currents were evoked by a stimulation pipette filled with extracellular solution located $100-300 \mu \mathrm{m}$ from the soma of the neuron being recorded from, usually near its primary apical dendrite. Care was taken to select inputs without detectable polysynaptic contributions and with minimal "jitter" in the timing of individual currents. The peak amplitude of the EPSCs was typically 10-15 times that of spontaneously occurring EPSCs. Voltage jumps from -70 to $-90 \mathrm{mV}$ were alternated with voltage jumps combined with synaptic stimulation. Jumps at different times relative to the onset of the conductance were randomized and interleaved to mitigate the effects of systematic changes in the experimental conditions over time (e.g., synaptic "rundown" or increases in series resistance). The stimulation rate was $0.25-0.33 \mathrm{~Hz}$.

Residual synaptic currents were obtained by subtracting the response to voltage jumps applied without synaptic stimulation from the response to jumps with stimulation. From 10 to 42 individual subtracted currents were averaged for each time point on the charge recovery curve (see Results). Synaptic charge was measured over an interval of 20-50 msec after the onset of the synaptic current. Sweeps that contained large spontaneous events were excluded from analysis. Charge recovery curves with the lowest noise levels were selected for analysis. Noise levels were quantified by dividing the SD of the fit residuals of the charge recovery curve by the difference between the maximum and minimum values of the fit curve; only complete charge recovery curves for which the value of this "noise index" was $\leq 0.11$ were accepted ( $n=8$ of 18 experiments). Statistical errors attributable to synaptic and instrumental noise were estimated by Monte Carlo simulation of synthetic charge recovery curves (Press et al., 1992). Gaussian noise (same noise index as the experimental charge recovery curves) was added to the charge recovery with mean experimental parameters. The resulting simulated charge recovery curves were fit by the same procedure as the experimental charge recovery curves. All values are given as mean \pm SEM.

\section{RESULTS}

\section{Attenuation and filtering of synaptic currents under poor space-clamp conditions}

The nature of the problem faced when attempting to voltage clamp dendritic synaptic currents via a somatic electrode is illustrated in Figure $1 B$ using the simple equivalent cylinder model shown in Figure $1 A$. There are two closely related components of inadequate space clamp that must be considered: attenuation of the signal along the cable, and the reduction in driving force at the synapse caused by local depolarization or hyperpolarization (also known as "voltage escape"). The outcome of these two effects is that the current recorded at the soma from synapses located on the dendrites is a substantially filtered version of the synaptic current expected under perfect clamp conditions, with the rise time, peak, and decay being subject to considerable distortion, dependent on the electrotonic distance of the synapse from the soma and the kinetics of the conductance. These features have been described in detail previously (Johnston and Brown, 1983; Rall and Segev, 1985; 


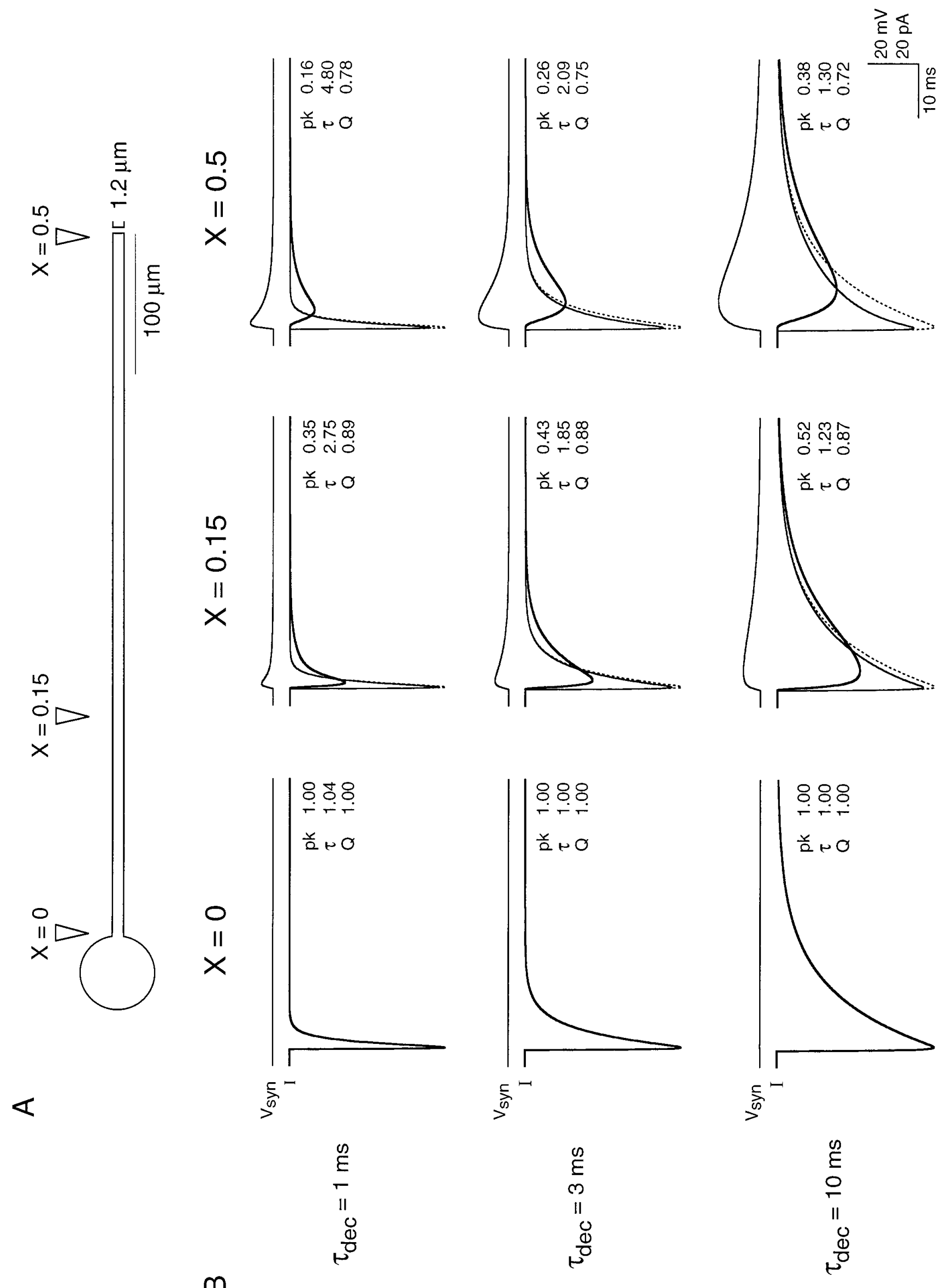


Major, 1993; Major et al., 1993; Spruston et al., 1993), but there are several aspects of particular relevance to the method that deserve special emphasis. First, the current flowing at the synapse during somatic voltage clamp is not identical to the current that would be flowing during perfect clamp of the synapse. This difference is attributable to the voltage escape at the synapse, which reduces the driving force of the synaptic current and distorts its shape. Second, for a given location and peak conductance the voltage escape, and thus the distortion of the synaptic current, is greatest for the synaptic conductances with the slowest kinetics, because they continue to charge the membrane capacitance for a longer period. The magnitude of this effect on the current recorded at the soma will be mitigated by the fact that slow conductances suffer less attenuation by the cable, because attenuation is frequency-dependent in a passive system (Rall, 1967; Jack et al., 1983; Spruston et al., 1994). Third, while the kinetics and the peak of the synaptic current suffer the most distortion, the attenuation of synaptic charge is much less severe. Furthermore, the attenuation of charge at a given location is relatively independent of the kinetics of the current; in these simulations, there was $<10 \%$ difference in the recovered charge for conductances with different kinetics even for the most distal synapses. This residual difference is attributable to the greater voltage escape caused by slower conductances: when the voltage escape converges toward zero, the attenuation of synaptic charge becomes independent of the kinetics of the synaptic conductance (Rall and Segev, 1985; Major et al., 1993).

The voltage jump method described in this paper circumvents the filtering of the synaptic current by the cable and provides a reliable estimate of the synaptic conductance time course for even the most electrotonically distal synapses. The method is particularly concerned with (and is most effective for) fast synaptic conductances, which suffer the most severe distortions under conditions of inadequate space clamp.

\section{Measuring charge recovery}

The experimental procedure for recovering synaptic charge, following the method introduced by Pearce (1993), is demonstrated using a simple equivalent cylinder simulation in Figure 2. According to this procedure the somatic voltage is held at the apparent synaptic reversal potential, and a hyperpolarizing voltage jump is made, providing a driving force to generate synaptic current. The voltage jump is repeated in the presence and absence of synaptic activation, and the resulting somatic currents are subtracted, thus eliminating the capacitive transient that accompanies the voltage jump. This procedure gives a residual synaptic current with a time course and amplitude that depend on the relative time of the jump and the onset of the synaptic conductance (see Fig. $3 A$ ). If the jump occurs sufficiently long before the onset of the conductance, then the residual current will approach identity with the synaptic current recorded at that potential under steady-state conditions. On the other hand, if the jump occurs a sufficiently long time after the onset of the synaptic conductance, then it will eventually recover no current at all, because the synaptic conductance will have terminated. The current resulting from each jump therefore results from voltage jump

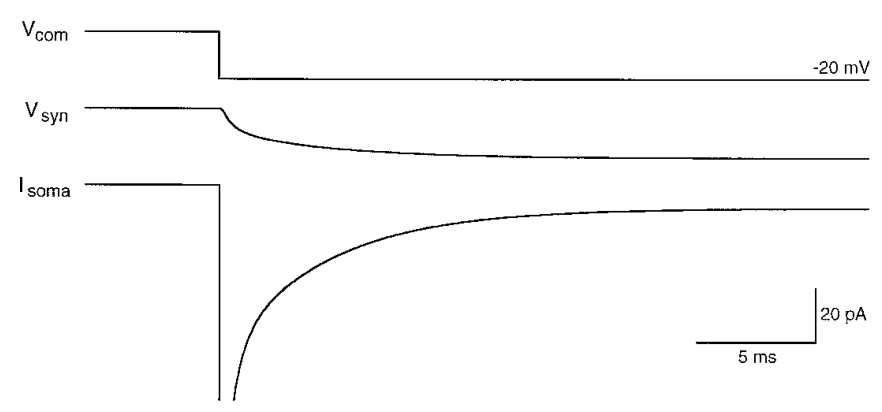

voltage jump with synaptic conductance

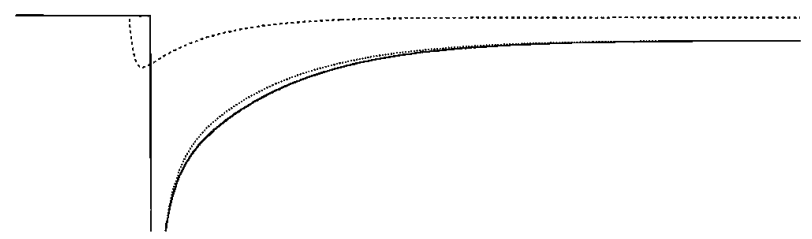

subtraction

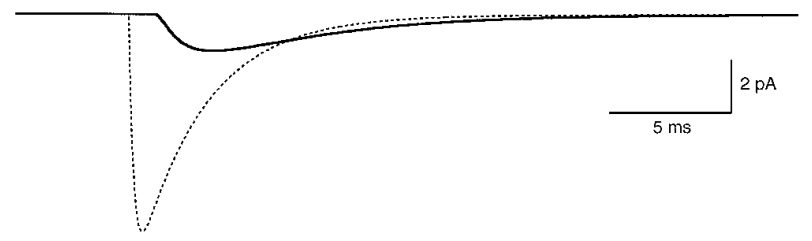

Figure 2. Experimental protocol for measuring charge recovery. Same equivalent cylinder as in Figure 1; synapse at $X=0.15$; peak conductance, $1 \mathrm{nS}$; rise and decay time constants, 0.2 and $3.0 \mathrm{msec}$, respectively. Top, $-20 \mathrm{mV}$ voltage jump applied at the soma via the somatic electrode. The somatic holding potential is set to $4.10 \mathrm{mV}$, making the voltage at the synapse equal to the reversal potential $(0 \mathrm{mV})$. The somatic voltage-clamp command is shown in the top trace; the voltage at the synapse is shown in the middle trace; and the (truncated) somatic clamp current is shown in the bottom trace. Middle, The synaptic conductance is activated $1 \mathrm{msec}$ before the same voltage jump. The time course of the synaptic conductance is shown by the dashed line, with the amplitude equal to that of the perfectly clamped synaptic current. The somatic clamp current in the presence (solid line) and absence (dotted line) of the synaptic conductance is shown. Bottom, Residual synaptic current (thick trace) after subtraction of somatic clamp current under the two conditions. The synaptic current expected under perfect voltage clamp at a constant holding potential of $-20 \mathrm{mV}$ is superimposed as a dashed line.

an interaction between the time course of the increase in driving force at the synapse and the kinetics of the conductance itself.

The synaptic charge associated with each residual current is plotted against the time of the respective jump in Figure $3 B$. The resulting "charge recovery curve" has a sigmoidal shape consisting of an exponential "onset" and "offset" with a transition at $\sim 0$

Figure 1. Space-clamp errors affecting the measurement of dendritic synaptic conductances. All traces in $B$ are from the same equivalent cylinder shown schematically in $A$ (soma not to scale), with $L=0.5$ and with synapses at three different electrotonic locations on the cable $(X=0,0.15$, and 0.5$)$. The peak synaptic conductance was $1 \mathrm{nS}$ in each case, consisting of the sum of a rising $(\tau=0.2 \mathrm{msec})$ and a decaying $(\tau=1,3$, or $10 \mathrm{msec})$ exponential. $B$, In each panel the voltage at the synaptic location $\left(V_{\mathrm{syn}}\right)$ is shown as the top trace. The bottom traces show the current recorded at the soma (thick line), the current actually flowing at the synapse (thin line), and the synaptic current expected under perfect voltage-clamp conditions (dashed line). The numbers at the right of each panel show the relative magnitude of the peak $(p k)$, the decay time constant $(\tau)$, and the charge $(Q)$ of the somatic current versus the perfectly clamped synaptic current. The scale bar at the bottom right applies to all panels. 
A
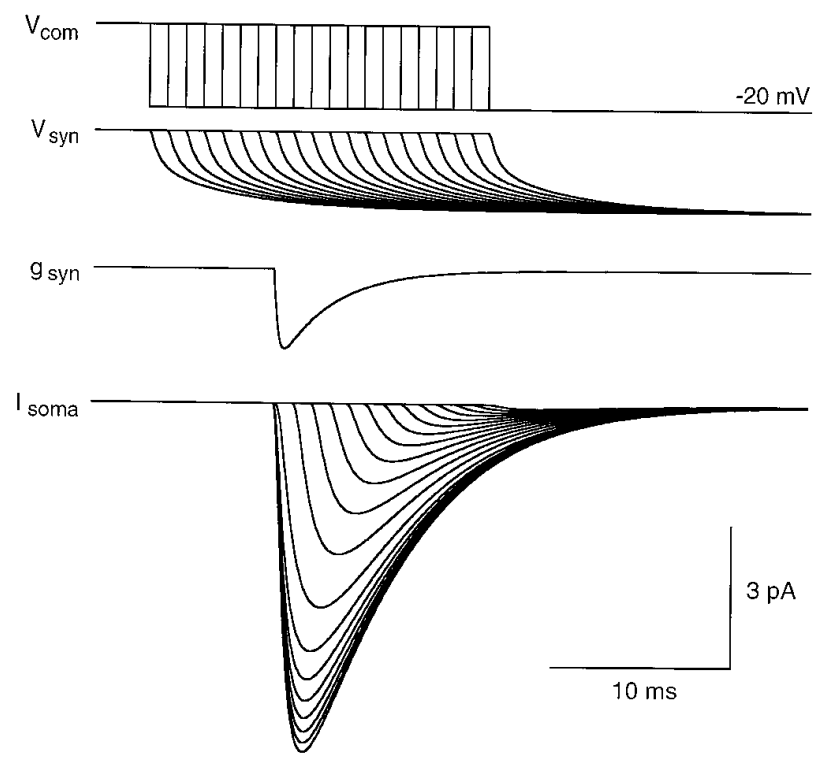

$\mathrm{B}$

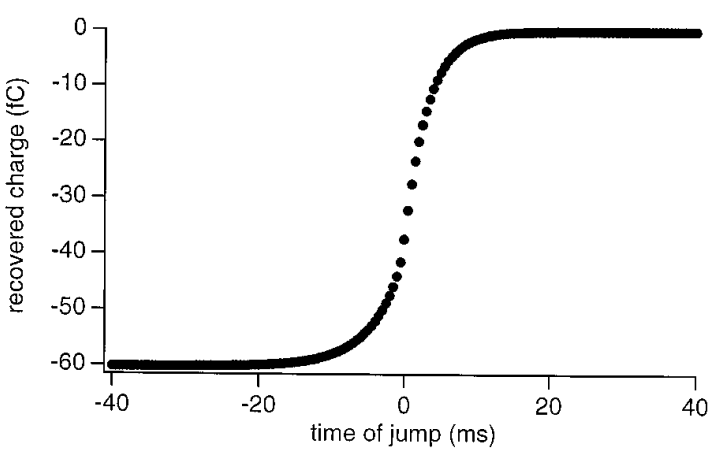

Figure 3. Charge recovery depends on the time of the voltage jump. Same conditions as in Figure 2. A, 20 superimposed sweeps of somatic voltage jumps ( $V_{\text {com }}$, top traces) at different times relative to the onset of the synaptic conductance. The interval between jump traces is $1 \mathrm{msec}$; the earliest jump is $7 \mathrm{msec}$ before the onset of the synaptic conductance, and the latest is $12 \mathrm{msec}$ after onset of the conductance. Also shown are the voltage at the synapse $\left(V_{\text {syn }}\right)$, the time course of the synaptic conductance $\left(g_{\text {syn }}\right)$, and the "recovered" somatic currents $\left(I_{\text {soma }}\right)$ obtained by subtracting the somatic clamp current in the presence and absence of the synaptic current for each jump. $B$, Plot of the charge associated with the recovered somatic synaptic currents $\left(I_{\text {soma }}\right)$ versus time of the somatic voltage jump; 0.5 msec jump intervals.

msec, i.e., at the beginning of the synaptic conductance. The determinants of the two components of the curve will be examined in the following section.

\section{Charge recovery after the onset of the synaptic conductance is determined by the conductance time course}

Figure 4 shows several charge recovery curves from a synapse at the same location as in Figures 2 and 3 with a range of kinetics for the synaptic conductance. It is clear from Figure 4 that the portion of the charge recovery curve that follows the onset of the synaptic conductance is determined by the decay time constant of the synaptic conductance; when the decay of the conductance is effectively instantaneous, as with the delta pulse, then no charge is recovered after $t=0 \mathrm{msec}$. For the more realistic synaptic conductances in Figure $4 B-D$, the decay of the charge recovery closely matches the actual decay time course of the synaptic conductance. This finding holds for the condition $\tau_{\text {rise }} \ll \tau_{\text {decay }}$ of the conductance, as is true for most synaptic conductances found to date. Generally, it was found that for a monoexponentially decaying synaptic conductance, the later the start time of the fit, the better the correspondence between the fit decay and actual decay, because starting the fit at later times helps avoid potential distortions attributable to voltage escape (see below). Of course, when the synaptic conductance time course is unknown it may be an oversimplification to assume that it has a single exponential decay (e.g., see Pearce, 1993).

\section{Charge recovery before the onset of the synaptic conductance is determined by the electrotonic distance of the synapse}

Figure 5 demonstrates that the early component of the charge recovery, before the onset of the synaptic conductance, reflects the time course of the voltage change at the synapse produced by the somatic voltage command. This was shown by placing a delta pulse synaptic conductance at various distances from the recording site, thereby eliminating the influence of synaptic kinetics on the charge recovery. Under these conditions, the charge recovery curve for a synapse located at the soma was essentially a step function, whereas the curve for more distal synapses became progressively more rounded. The same was true for the voltage response to a somatic voltage jump at different distances. The symmetry between the time course of the two curves is demonstrated by overlaying the scaled voltage response on top of the charge recovery, as shown in Figure $5 D$.

\section{A simple analytical function describes the charge recovery curve}

In a linear system, the voltage response at the synapse to a somatic voltage step can always be described by a sum of exponentials (Rall, 1969; Major et al., 1993) [we follow the convention of Major et al. (1993) in setting resting membrane potential and the reversal potential of the synaptic conductance to zero]. This sum is often dominated by a single exponential, with time constant $\tau_{\mathrm{v}}$ (see Fig. $3 A$ ):

$$
V_{\text {syn }}(s, t)=\alpha V_{\text {com }} \Theta(t-s)\left(1-\mathrm{e}^{-(t-s) / \tau_{\mathrm{v}}}\right)
$$

Figure 4. Charge recovery after the onset of the synaptic conductance is determined by the synaptic decay. $A-D$, Charge recovery plots for synaptic conductances with different kinetics: a delta pulse $(A)$ or a double-exponential function with the same rising exponential $(0.2 \mathrm{msec})$ and different decay time constants $(1,3$, and $10 \mathrm{msec}$ in $B-D$, respectively). Peak conductance $1 \mathrm{nS}$ in each case; all synapses were located at $X=0.15$ using the same equivalent cylinder as in Figure 1. A single-exponential decay has been fit to the decay of the charge recovery in $B-D$; note the close correspondence with the decay time constant $\left(\tau_{\mathrm{dec}}\right)$ of the original synaptic conductance in each case. $E$, Each charge recovery curve has been normalized by its value at the onset of the synaptic conductance and superimposed. The individual points of each curve have been joined by a line for clarity. 


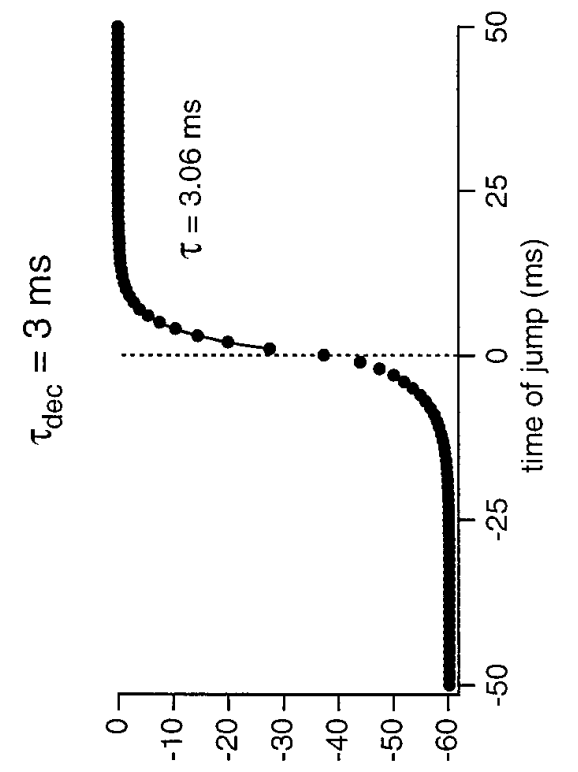

(ว) әอлец八 рәләлорәл

0

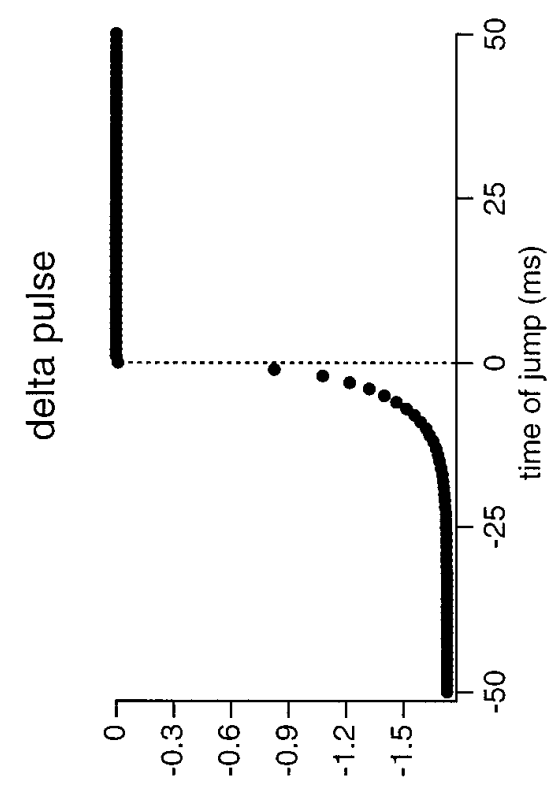

(О) әбдецว рәләлорәд

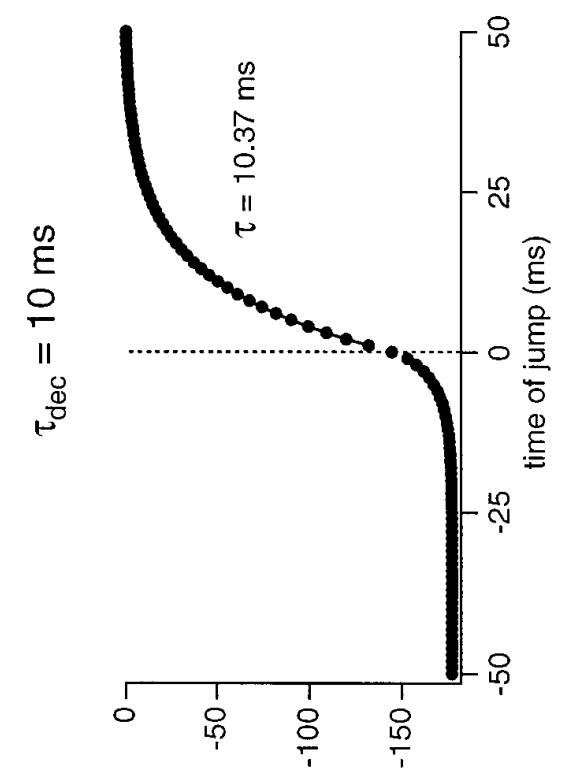

(О) әอдецо рәләлоэәд

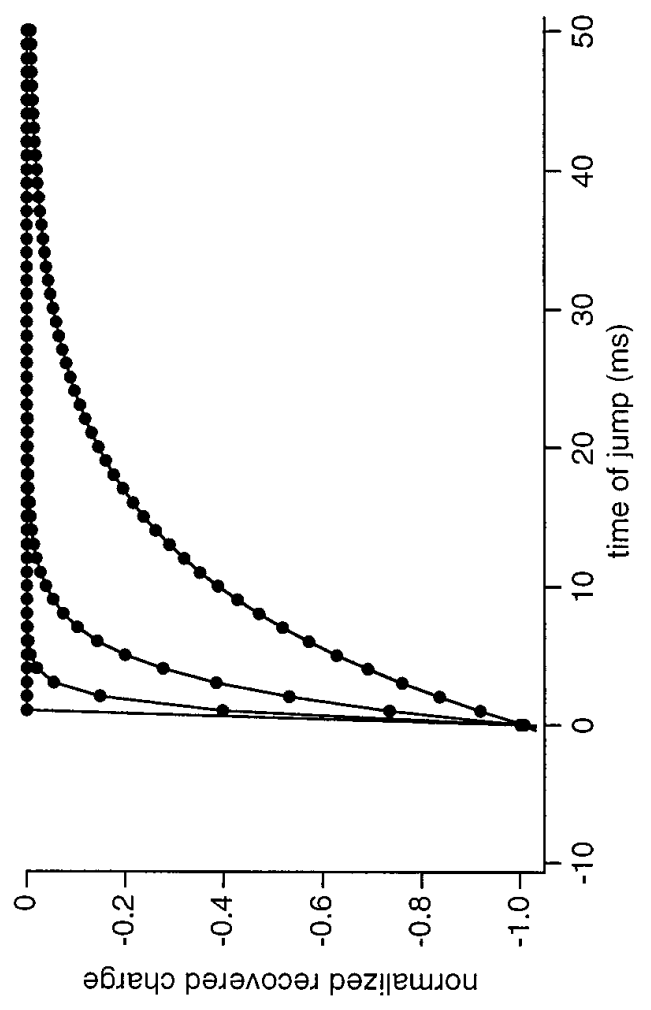

$\varangle$

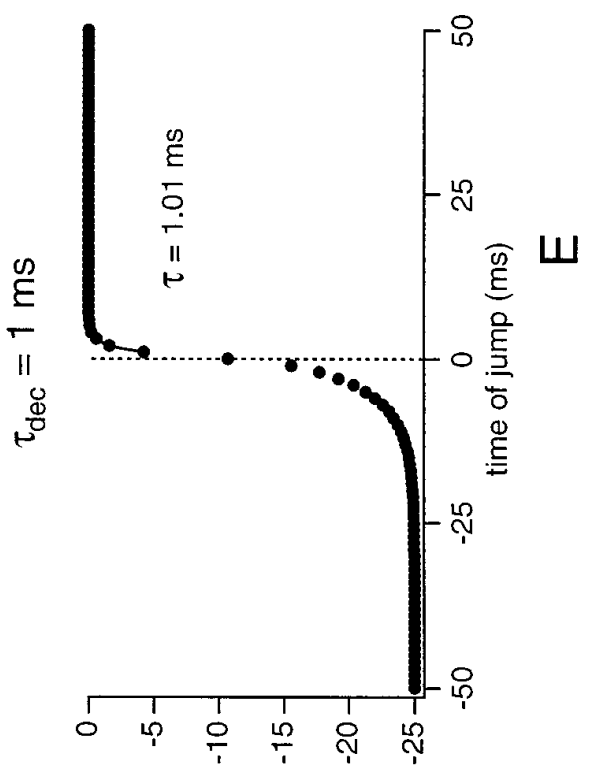

(О) әб.ечо рәләлорәд 


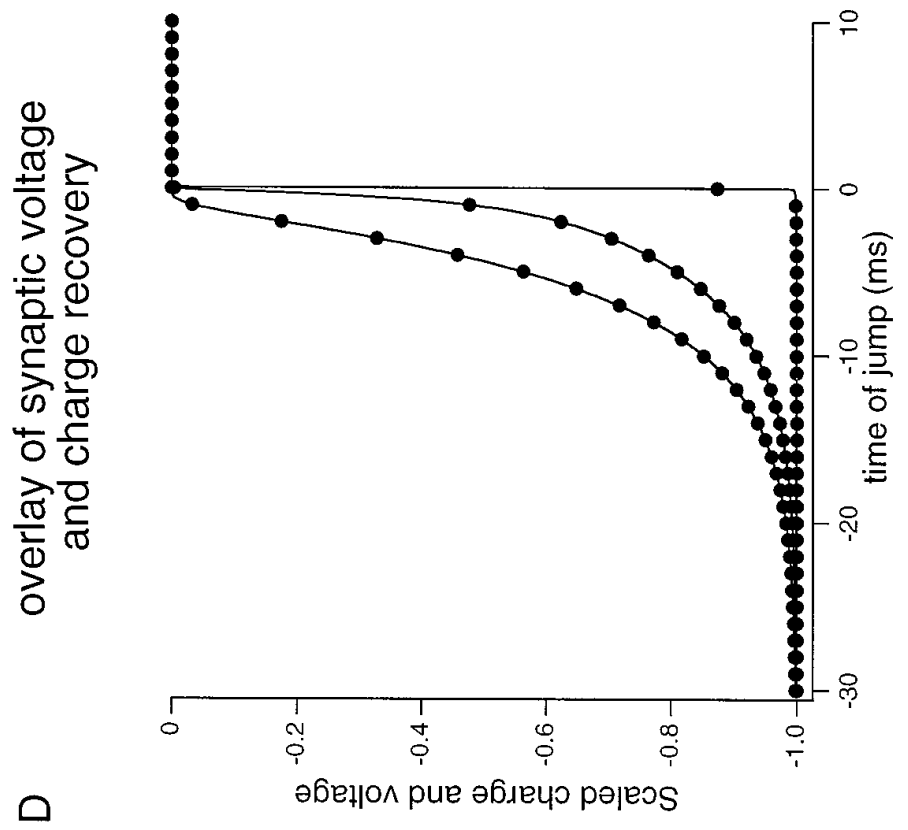

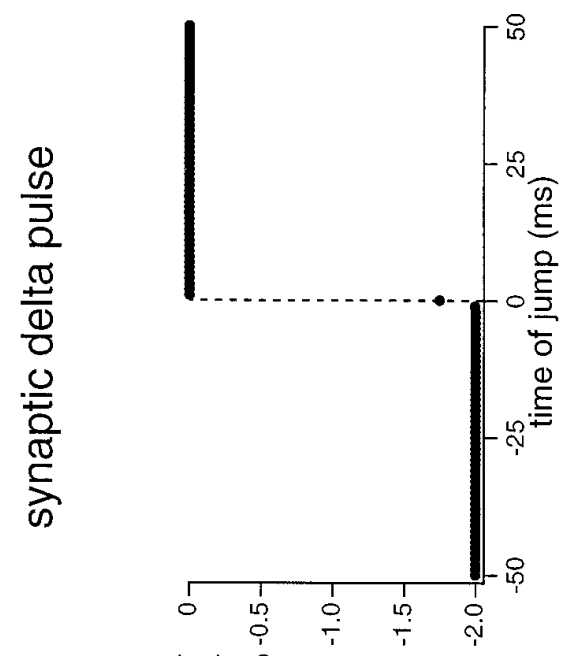

○ (Оt) әблечо рәләлоэәл II

$\frac{\frac{\rho}{\Phi}}{\infty}$

(1)

完

$\leftarrow$

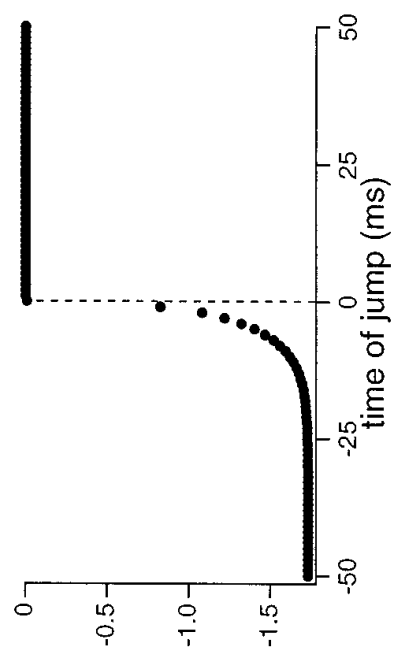

(О) әблечо рәләлооәл

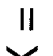

$\times$

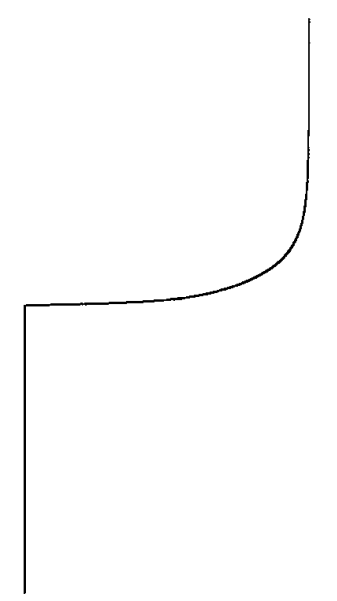

$\sum^{5}$
L?

II

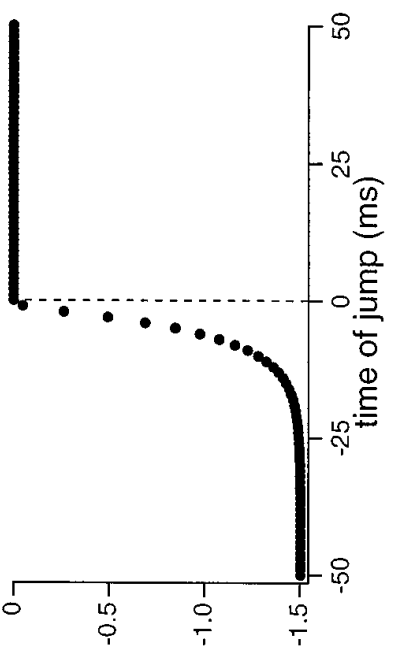

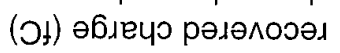

$\times$

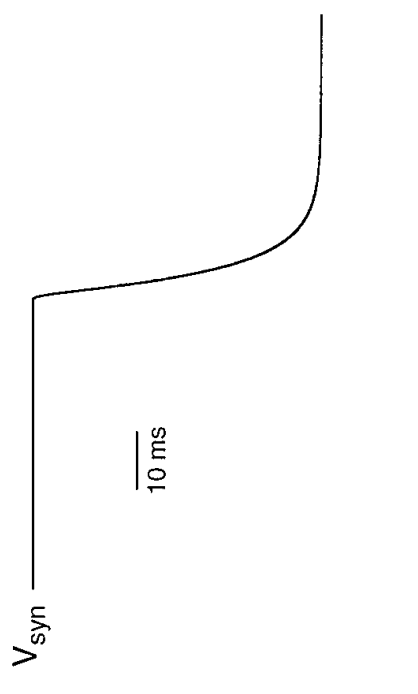


where $\alpha$ is the steady-state attenuation factor of the voltage command, $V_{\text {com }}$, at the soma, $\Theta$ is the Heaviside step function:

$$
\Theta(x)= \begin{cases}1 & (x \geq 0) \\ 0 & (x<0)\end{cases}
$$

and $s$ is the time of the voltage step with respect to the onset ( $t=$ $0)$ of the synaptic conductance, $g(t)$.

For simplicity, we first choose a $\delta$ function synaptic conductance:

$$
g(t)=\hat{g} \delta(t) .
$$

The resulting current flowing at the synapse (neglecting voltage escape):

$$
I_{\text {syn }}(s, t)=V_{\text {syn }}(s, t) g(t)
$$

can be integrated over time to give the synaptic charge:

$$
Q_{\text {syn }}(s)=\int_{-\infty}^{\infty} I_{\text {syn }}(s, t) \mathrm{d} t=\alpha \mathrm{V}_{c o m} \hat{g} \Theta(-s)\left(1-e^{s / \tau_{\mathrm{v}}}\right),
$$

which of course depends on the time of jump, $s$ (see Fig. $3 B$ ). The charge recovered at the somatic voltage clamp electrode:

$$
Q_{\text {soma }}(s)=\alpha^{2} V_{\operatorname{com}} \hat{g} \Theta(-s)\left(1-e^{s / \tau_{\mathrm{v}}}\right)
$$

is a constant fraction $\alpha$ of the total synaptic charge (Redman, 1973; Rinzel and Rall, 1974; Carnevale and Johnston, 1982; Jack et al., 1983; Rall and Segev, 1985; Major et al., 1993).

The assumption of a $\delta$ function synaptic conductance is unrealistic, and therefore we repeat the calculation in Equation 4 with a synaptic conductance that rises instantaneously to a peak at $t=$ 0 and then decays exponentially with time constant $\tau_{\mathrm{dec}}$ :

$$
g(t)=\bar{g} \Theta(t) e^{-t / \tau_{\mathrm{dec}}}
$$

which yields a recovered charge:

$$
Q_{\text {soma }}(s)=\left\{\begin{array}{cc}
\frac{\alpha^{2} V_{\mathrm{com}} \bar{g} \tau_{\mathrm{dec}}\left(\tau_{\mathrm{dec}}+\tau_{\mathrm{v}}\left(1-e^{s / \tau_{\mathrm{v}}}\right)\right)}{\tau_{\mathrm{dec}}+\tau_{\mathrm{v}}} & (s \leq 0) \\
\frac{\alpha^{2} V_{\mathrm{com}} \bar{g} \tau_{\mathrm{dec}}^{2} e^{-s / \tau_{\mathrm{dec}}}}{\tau_{\mathrm{dec}}+\tau_{\mathrm{v}}} & (s>0)
\end{array}\right.
$$

that changes exponentially with a single time constant equal to $\tau_{\mathrm{v}}$ for voltage jumps occurring before the onset of the synaptic conductance and a single time constant equal to $\tau_{\mathrm{dec}}$ afterward (compare Figs. 5 and 4). The ratio of the amplitudes of the onset and offset phases of the charge recovery is equal to $\tau_{\mathrm{v}} / \tau_{\mathrm{dec}}$ Because integration is a linear operation, the integral in Equation 4 can still be evaluated if both the voltage response at the synapse and the synaptic conductance are described by sums of exponentials. The time constants of the charge recovery for $s \leq 0$ are given by the time constants of the voltage response, and the time constants of the charge recovery for $s>0$ are given by the time constants of the synaptic conductance. We illustrate this for the case that the voltage response at the synapse is a sum of two exponentials:

$$
V_{\mathrm{syn}}(s, t)=\alpha V_{\mathrm{com}} \Theta(t-s)\left(a_{\mathrm{v}_{1}}+a_{\mathrm{v}_{2}}-a_{\mathrm{v}_{1}} e^{-(t-s) / \tau_{\mathrm{v}}}-a_{\mathrm{v}_{2}} e^{-(t-s) / \tau_{\mathrm{v}}}\right),
$$

and the synaptic conductance is represented by three exponentials (one for the rise and two for the decay):

$$
g(t)=\Theta(t)\left(-\left(\bar{g}_{1}+\bar{g}_{2}\right) e^{-t / \tau_{\mathrm{rise}}}+\bar{g}_{1} e^{-t / \tau_{\mathrm{dec}}}+\bar{g}_{2} e^{-t / \tau_{\mathrm{dec}}}\right) .
$$

In this case the recovered charge is:

$$
\begin{aligned}
& Q_{\text {soma }}(s \leq 0)= \\
& \alpha^{2} V_{\operatorname{com}}\left(\left(a_{\mathrm{v}_{1}}+a_{\mathrm{v}_{2}}\right)\left(-\left(\bar{g}_{1}+\bar{g}_{2}\right) \tau_{\text {rise }}+\bar{g}_{1} \tau_{\mathrm{dec}_{1}}+\bar{g}_{2} \tau_{\mathrm{dec}_{2}}\right)\right. \\
& +a_{\mathrm{v}_{1}} \tau_{\mathrm{v}_{1}} e^{s / \tau_{\mathrm{v}_{1}}}\left(\frac{\left(\bar{g}_{1}+\bar{g}_{2}\right) \tau_{\mathrm{rise}}}{\tau_{\mathrm{rise}}+\tau_{\mathrm{v}_{1}}}-\frac{\bar{g}_{1} \tau_{\mathrm{dec}_{1}}}{\tau_{\mathrm{dec}_{1}}+\tau_{\mathrm{v}_{1}}}-\frac{\bar{g}_{2} \tau_{\mathrm{dec}_{2}}}{\tau_{\mathrm{dec}_{2}}+\tau_{\mathrm{v}_{1}}}\right) \\
& \left.+a_{\mathrm{v}_{2}} \tau_{\mathrm{v}_{2}} e^{s / \tau_{\mathrm{v}_{2}}}\left(\frac{\left(\bar{g}_{1}+\bar{g}_{2}\right) \tau_{\mathrm{rise}}}{\tau_{\mathrm{rise}}+\tau_{\mathrm{v}_{2}}}-\frac{\bar{g}_{1} \tau_{\mathrm{dec}_{1}}}{\tau_{\mathrm{dec}_{1}}+\tau_{\mathrm{v}_{2}}}-\frac{\bar{g}_{2} \tau_{\mathrm{dec}_{2}}}{\tau_{\mathrm{dec}_{2}}+\tau_{\mathrm{v}_{2}}}\right)\right) \\
& Q_{\text {soma }}(s>0)= \\
& \alpha^{2} V_{\text {com }}\left(\left(a_{\mathrm{v}_{1}}+a_{\mathrm{v}_{2}}\right)\left(-\left(\bar{g}_{1}+\bar{g}_{2}\right) \tau_{\mathrm{rise}} e^{-s / \tau_{\mathrm{rise}}}+\bar{g}_{1} \tau_{\mathrm{dec}_{1}} e^{-s / \tau_{\mathrm{dec}}}+\bar{g}_{2} \tau_{\mathrm{dec}_{2}} e^{-s / \mathrm{dec}_{2}}\right)\right. \\
& +a_{\mathrm{v}_{1}} \tau_{\mathrm{v}_{1}}\left(\frac{\left(\bar{g}_{1}+\bar{g}_{2}\right) \tau_{\mathrm{rise}} e^{-s / \tau_{\mathrm{rise}}}}{\tau_{\text {rise }}+\tau_{\mathrm{v}_{1}}}-\frac{\bar{g}_{1} \tau_{\mathrm{dec}_{1}} e^{-s / \tau_{\mathrm{dec}}}}{\tau_{\mathrm{dec}_{1}}+\tau_{\mathrm{v}_{1}}}-\frac{\bar{g}_{2} \tau_{\mathrm{dec}_{2}} e^{-s / \tau_{\mathrm{dec}}}}{\tau_{\mathrm{dec}_{2}}+\tau_{\mathrm{v}_{1}}}\right) \\
& \left.+a_{\mathrm{v}_{2}} \tau_{\mathrm{v}_{2}}\left(\frac{\left(\bar{g}_{1}+\bar{g}_{2}\right) \tau_{\text {rise }} e^{-s / \tau_{\mathrm{rise}}}}{\tau_{\mathrm{rise}}+\tau_{\mathrm{v}_{2}}}-\frac{\bar{g}_{1} \tau_{\mathrm{dec}_{1}} e^{-s / \tau_{\mathrm{dec}}}}{\tau_{\mathrm{dec}_{1}}+\tau_{\mathrm{v}_{2}}}-\frac{\bar{g}_{2} \tau_{\mathrm{dec}_{2}} e^{-s / \tau_{\mathrm{dec} 2}}}{\tau_{\mathrm{dec}_{2}}+\tau_{\mathrm{v}_{2}}}\right)\right) \text {. }
\end{aligned}
$$

To allow well conditioned fits of charge recovery data, the amplitudes $a_{\mathrm{v}_{1}}$ and $a_{\mathrm{v}_{2}}$ in Equation 10 were normalized according to $a_{\mathrm{v}_{1}}$ $+a_{\mathrm{v}_{2}}=1$. The factors $\alpha^{2}, V_{\text {com }}, \bar{g}_{1}$ and $\bar{g}_{2}$ were combined in two overall amplitudes of the fit function, $g_{1}^{*}=\alpha^{2} V_{\text {com }} \bar{g}_{1}$ and $g_{2}^{*}=$ $\alpha^{2} V_{\text {com }} \bar{g}_{2}$, which were free parameters of the fit. Constant offsets in $s$ and $Q_{\text {soma }}(s)$ can also be introduced to allow latency variations and jumps from other potentials than the apparent reversal potential of the synaptic conductance.

In practice it may not always be necessary (or possible) to fit the entire analytical function. As demonstrated above, the charge recovery can be separated into two components, with the second determined by the kinetics of the conductance (see Fig. 4 and Eqs. 7 and 10). This can be exploited experimentally in situations in which the time of recording is limited or in which only the decay of the synaptic conductance is of interest. By making a series of jumps at different times after the onset of the synaptic conductance and then fitting the decay of the recovered charge with an exponential function, an estimate can be made of the decay of the conductance (assuming that $\tau_{\text {rise }} \ll \tau_{\text {decay }}$ ). It is also possible to fit multiple exponential functions to the decay; in this case, the

Figure 5. Charge recovery before the onset of the synaptic conductance reflects the voltage change at the synapse caused by the somatic voltage command. All simulations are from the same equivalent cylinder as in Figure 1 . A-C, Left panels, Synaptic voltage $\left(V_{\text {syn }}\right)$ in response to a somatic voltage-clamp step (of arbitrary amplitude) at three different locations; right panels, charge recovery curves for a synaptic delta pulse (1 nS peak conductance) at the same three locations. $D$, Superimposition of the synaptic voltage responses on the respective charge recoveries; both the charge recoveries and the voltage responses have been normalized by their respective maxima, and the time axis of the voltage response has been inverted. Note the exact correspondence of the voltage time course and the charge recovery in each case. 
A

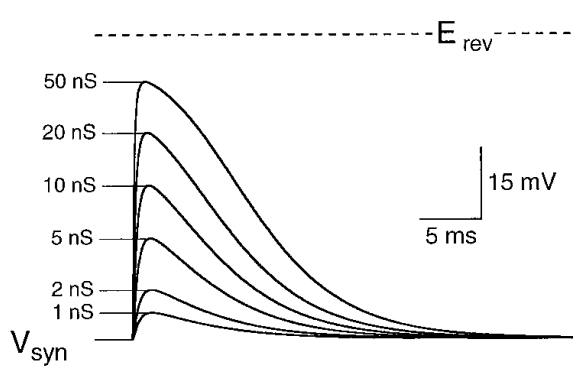

B

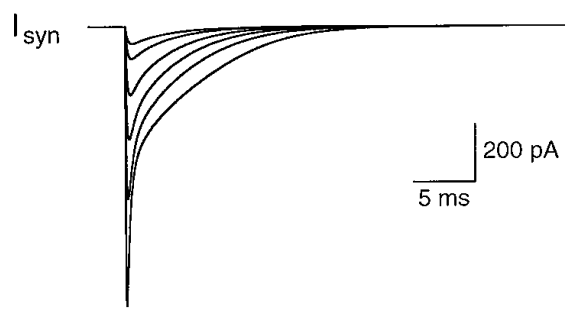

C

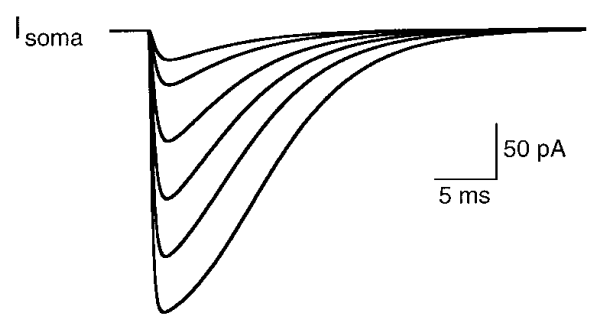

D

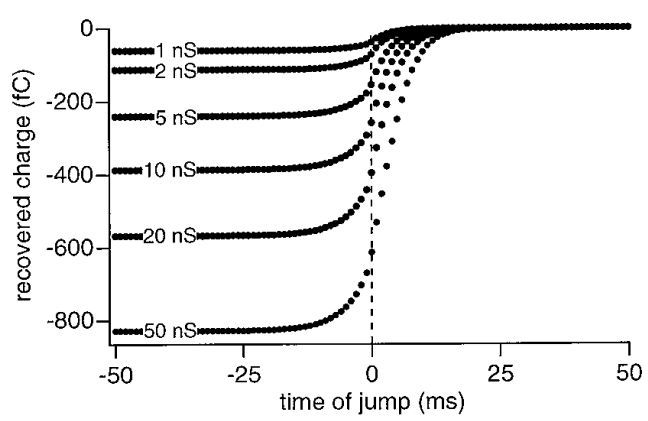

$E$

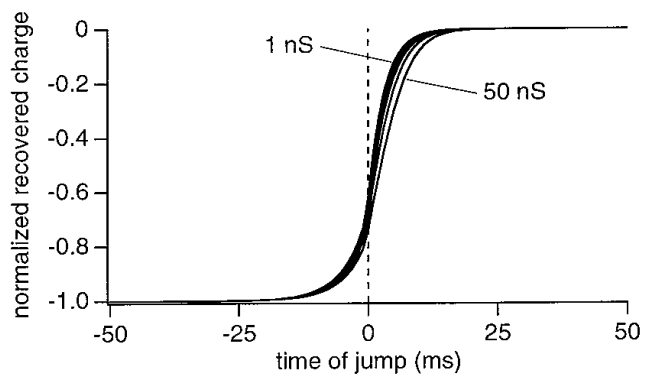

$\mathrm{F}$

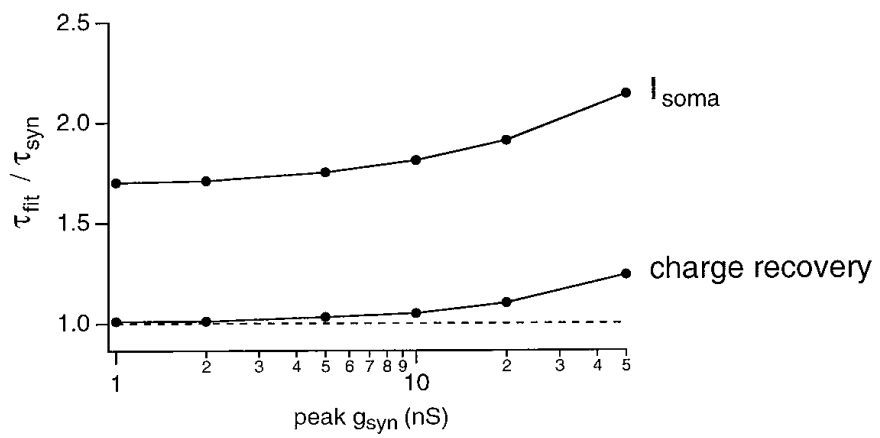

Figure 6. Effects of local depolarization (voltage escape) on the reliability of the charge recovery method. $A-C$, Simulations from the same equivalent cylinder as in Figure 1, with the synapse at a constant location $(X=0.15)$ and with a range of peak synaptic conductances as indicated (rising and decaying time constants, 0.2 and $3.0 \mathrm{msec}$ respectively). $A$, Voltage at the synapse $\left(V_{\mathrm{syn}}\right) ; B$, current flowing at the synapse $\left(I_{\text {syn }}\right) ; C$, Current recorded at the soma $\left(I_{\text {soma }}\right)$. The charge recovery plots from the various conductances are shown unscaled in $D$ and scaled by the peak charge in $E$. The graph in $F$ compares the decay time constant obtained by fitting either the somatic current or the charge recovery curve $\left(\tau_{\text {fit }}\right.$; fit beginning 7 msec after onset of the conductance in each case) with the actual decay time constant of the synaptic conductance $\left(\tau_{\text {syn }}\right)$. Note that the time constant estimated by the charge recovery is relatively faithful to the actual synaptic decay time constant except at very high values of peak conductance.

time constants will be extracted faithfully, but the relative amplitudes of the faster components will be underestimated. This "shortcut" could in principle allow the voltage jump method to be applied to spontaneous synaptic currents, by triggering voltage jumps (using a software or hardware trigger) with a variable delay after the synaptic current crosses a threshold amplitude. Some jitter will be introduced in the time of the jump if the spontaneous currents have widely different amplitudes and/or rise times; this

Figure 7. Simulations of the voltage jump method in a CA3 pyramidal cell model. $A$, Morphology of the CA3 pyramidal cell with which the simulations were performed showing the location of the simulated synapse, which was placed on a spine head ( filled circle). $B-G$, A synaptic conductance (peak, 0.5 $\mathrm{nS}$ ) consisting of a double-exponential function $\left(\tau_{\text {rise }}=0.2 \mathrm{msec} ; \tau_{\mathrm{dec}}=2.5 \mathrm{msec}\right)$ was used; the conditions in $B-D$ and $E-G$ are identical, except that the series resistance of the somatic pipette was $0.5 \mathrm{M} \Omega$ in $B-D$ and $20 \mathrm{M} \Omega$ in $E-G$. $B, E$, Somatic clamp current resulting from activation of the synaptic conductance (thick trace) as well as the synaptic current expected under conditions of perfect space clamp. The $20-80 \%$ rise times of the currents were $1.00 \mathrm{msec}$ in $B$ and $1.71 \mathrm{msec}$ in $E$. The decay time course of the somatic clamp current could be fit with a single exponential function with $\tau=6.44 \mathrm{msec}$ in $B$ and $12.90 \mathrm{msec}$ in $E$. $C, F$, Currents recovered by a series of $-20 \mathrm{mV}$ voltage jumps from $-65 \mathrm{mV}(1 \mathrm{msec}$ interval between jumps). $D, G$, Charge recovery curves measured from the traces in $C$ and $F$ together with the best fit of the analytical function (Eq. 10). Note the different onset of the two curves. For the low series resistance condition the best fit was with the following parameters: $\tau_{\mathrm{v}_{1}}=1.58 \mathrm{msec}(40 \%) ; \tau_{\mathrm{v}_{2}}=8.53 \mathrm{msec}(60 \%) ; \tau_{\text {rise }}=0.22$ msec; and $\tau_{\mathrm{dec}}=2.55 \mathrm{msec}$ (here and wherever appropriate, Eq. 10 was modified such that $\tau_{\mathrm{dec}_{1}}=\tau_{\mathrm{dec}_{2}}=\tau_{\mathrm{dec}}$ ). For the high series resistance condition the best fit was with $\tau_{\mathrm{v}_{1}}=2.15 \mathrm{msec}(5 \%) ; \tau_{\mathrm{v}_{2}}=12.75 \mathrm{msec}(95 \%) ; \tau_{\text {rise }}=0.19 \mathrm{msec}$; and $\tau_{\mathrm{dec}}=2.56 \mathrm{msec}$. A single-exponential fit to the decay of the charge recovery curve gave $\tau_{\mathrm{dec}}=2.54 \mathrm{msec}$ in both cases. $H-J$, An NMDA receptor-mediated synaptic conductance was simulated at the same location (peak, $0.1 \mathrm{nS} ; \tau_{\text {rise }}=5.0 \mathrm{msec} ; \tau_{\text {dec }}=40 \mathrm{msec}$ ) with $0.5 \mathrm{M} \Omega$ series resistance, assuming zero external $\mathrm{Mg}^{2+} . H$ compares the perfectly clamped synaptic current with the measured somatic current. The $20-80 \%$ rise time of the somatic current in $H$ was 7.17 msec, and current was fit with a double-exponential function with $\tau_{\text {rise }}=9.6 \mathrm{msec}$ and $\tau_{\mathrm{dec}}=39.8 \mathrm{msec}$. The currents recovered by voltage jumps from -65 to $-85 \mathrm{mV}$ are shown in $I$, and the respective charge recovery curve is shown in $J$. The values of the best fit of the analytical function were $\tau_{\mathrm{v}_{1}}=1.45 \mathrm{msec}(41 \%) ; \tau_{\mathrm{v}_{2}}=8.58 \mathrm{msec}$ $(59 \%) ; \tau_{\text {rise }}=5.15 \mathrm{msec}$; and $\tau_{\mathrm{dec}}=40.4 \mathrm{msec}$. A single-exponential fit to the decay of the charge recovery curve gave $\tau_{\mathrm{dec}}=41.2 \mathrm{msec}$. 


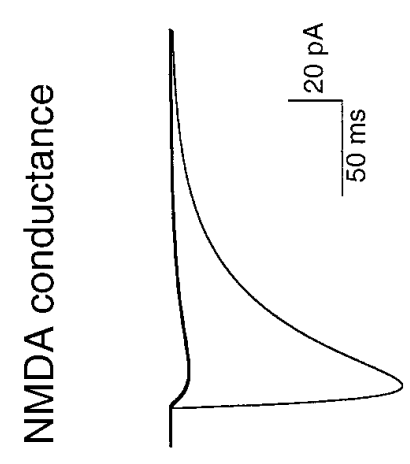

I

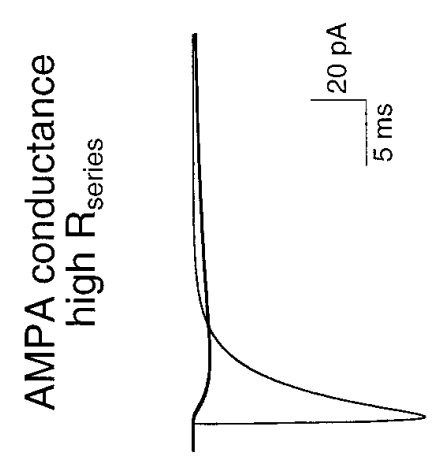

ш

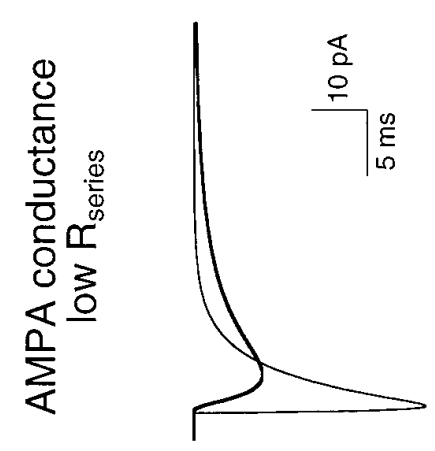

$\infty$
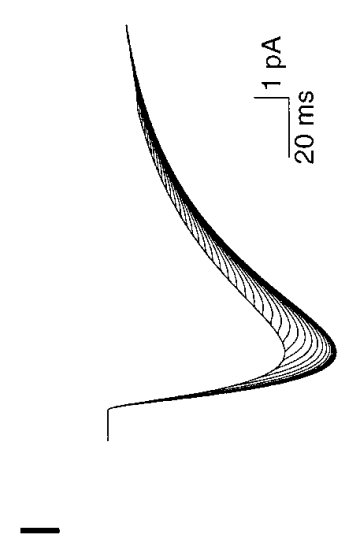

山
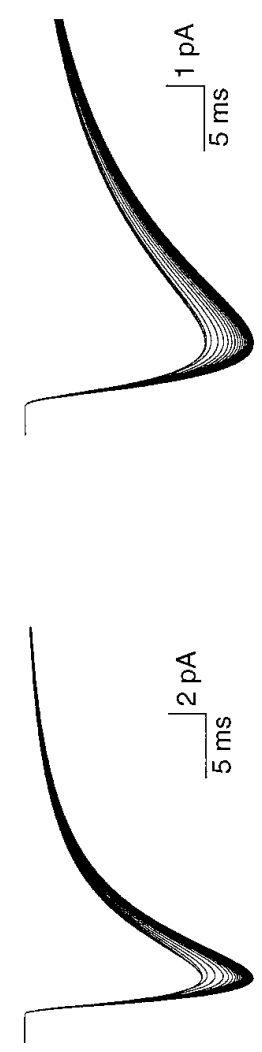

0

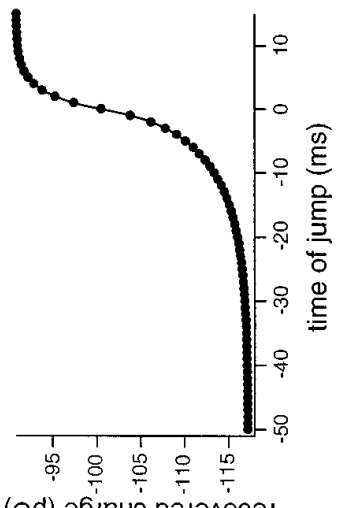

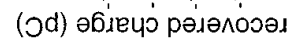

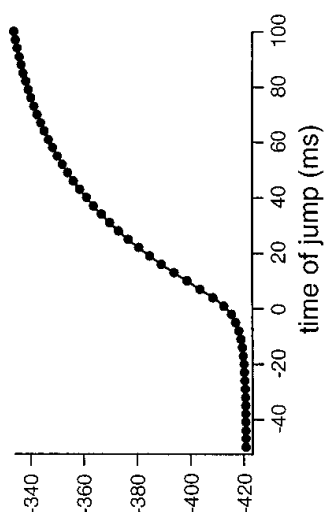

$\longrightarrow \quad$ (od) әБцецо рәләлорәд

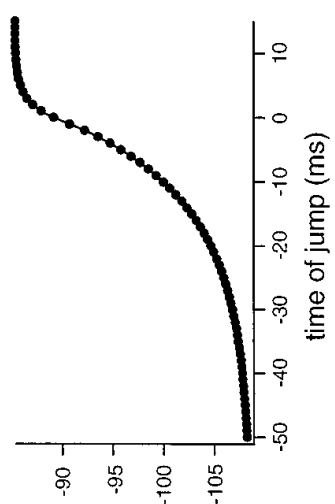

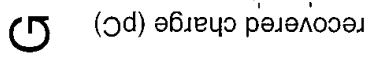

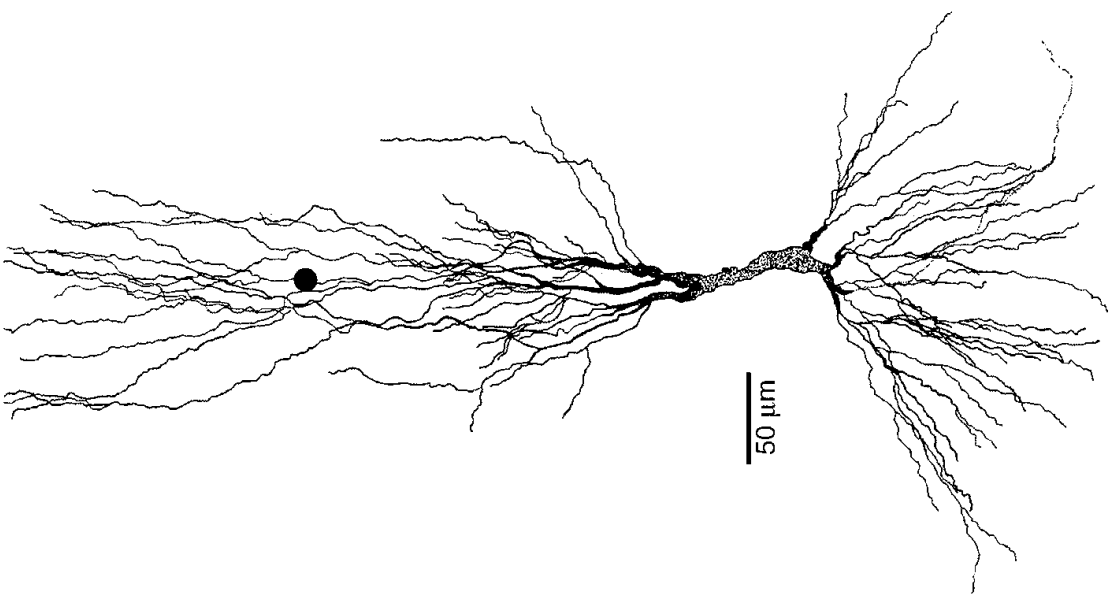




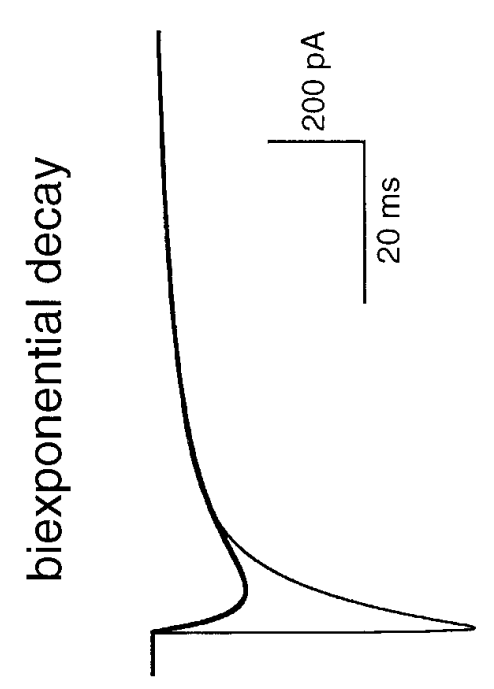

Ш

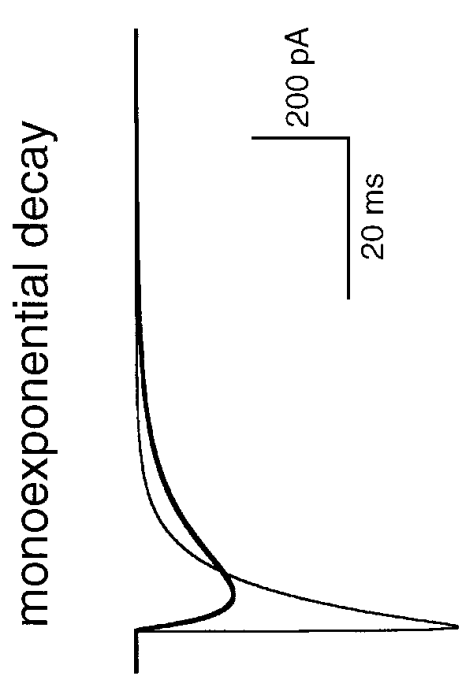

$m$

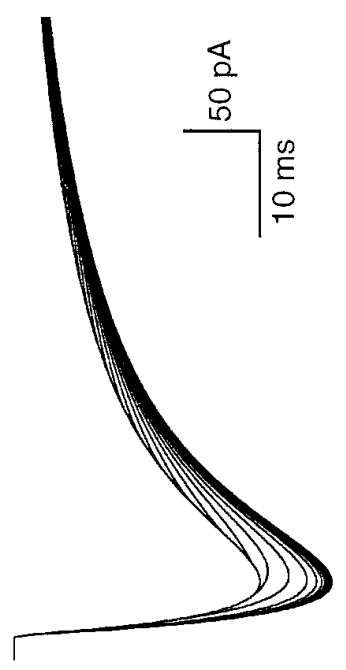

レ

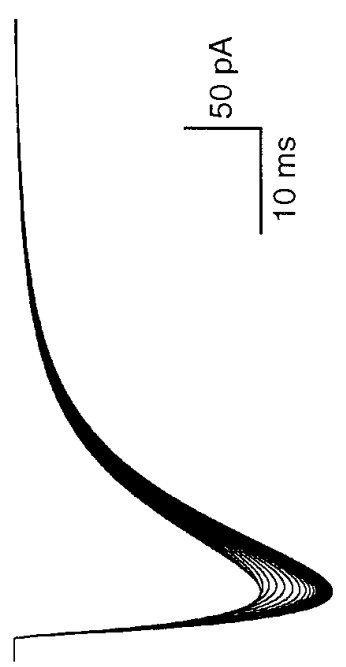

0

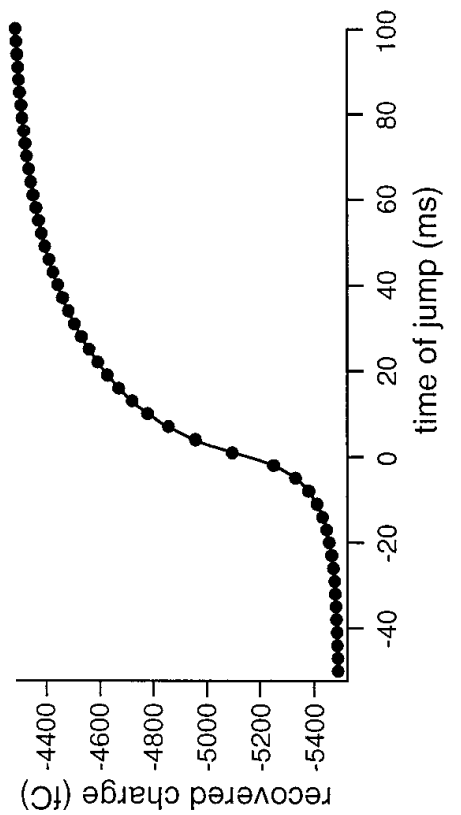

v
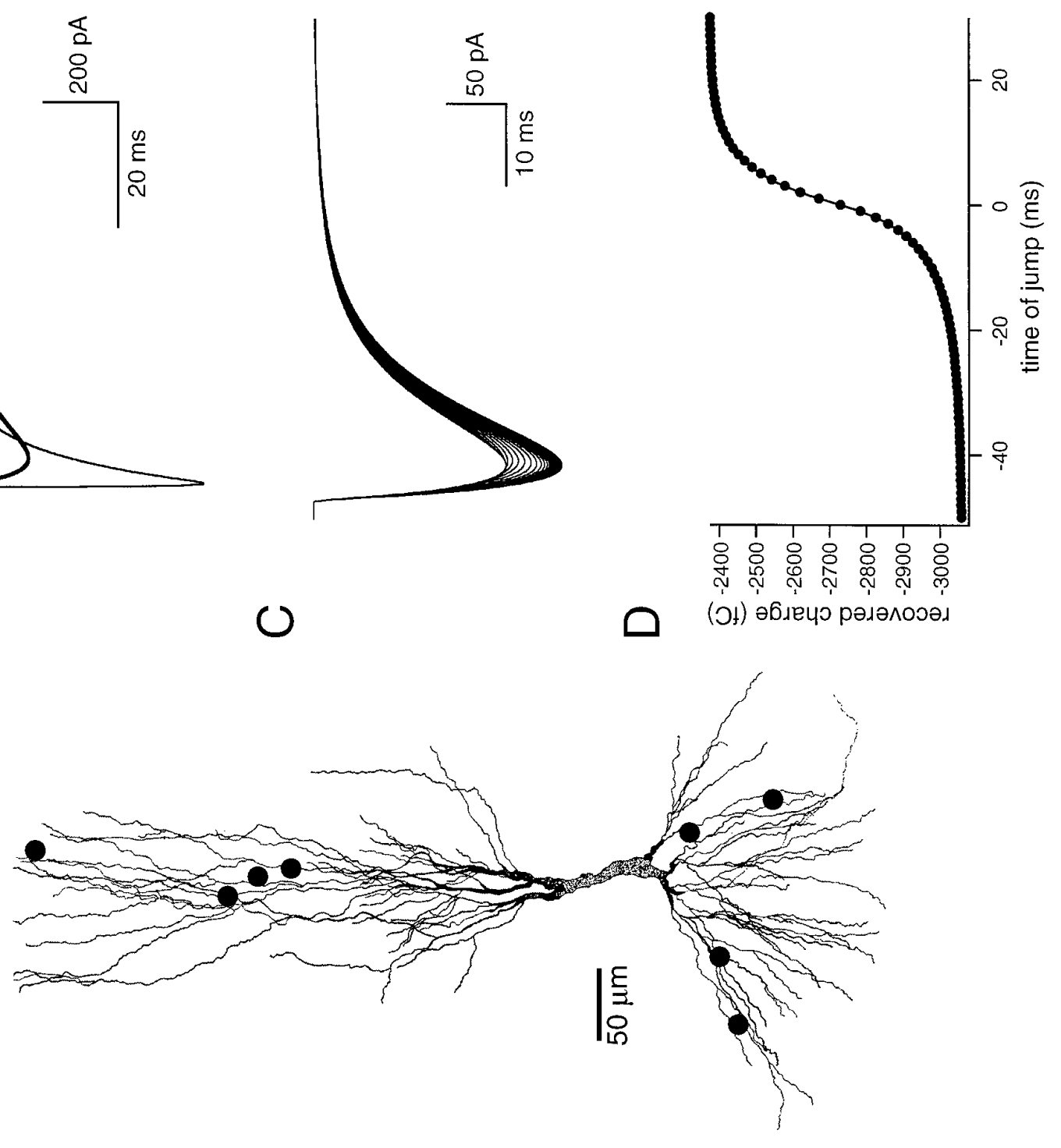

$\varangle$ 
can be corrected for by later normalizing the time of each jump to a reference point on the rise. As with evoked synaptic conductances, the mean decay time course of the underlying conductances can then be estimated from the charge recovery curve.

\section{The voltage jump method also works in current-clamp mode}

In principle, a change in driving force at the synapse can be generated either with a voltage command under voltage clamp or by injecting a fixed amount of current to generate a reproducible voltage change in current-clamp mode. Because the analytical solutions for both the "time integral" of synaptic potentials and the synaptic charge in voltage clamp depend only on the charge flowing at the synapse (Major et al., 1993), one can fit the curve of the time integral of the synaptic potentials obtained after a series of identical square current pulses with Equation 7 or 10. In this case, the measured $\tau_{\mathrm{v}}$ will be determined by the membrane time constant $\tau_{\mathrm{m}}$, because $\tau_{\mathrm{m}}$ determines the dendritic voltage response to a square current step (neglecting the faster equalization time constants, which generally have much smaller amplitudes for a long current step). Although the kinetics of the synaptic conductance can be extracted reliably as described above, because $\tau_{\mathrm{m}} \gg \tau_{\text {decay }}$ for most neurons and synaptic conductances, the amplitude of the time integral curve will be dominated by the component attributable to $\tau_{\mathrm{m}}$ (the onset). Therefore, for determining the time course of the synaptic conductance it is always preferable to use voltage clamp rather than current clamp, because $\tau_{\mathrm{v}}$ for voltage clamp will always be smaller than $\tau_{\mathrm{m}}$ [except in the limiting case, in which they are identical (Major et al., 1993)] and thus will provide better signal-to-noise ratios for extracting $\tau_{\text {rise }}$ and $\tau_{\text {decay }}$. Voltage clamp will also reduce the voltage excursion at the synapse (although only slightly for some synapses) and thus also distortion in the synaptic current. For these reasons all subsequent simulations as well as the experiments were done in voltage-clamp mode.

\section{Effect of voltage escape at the synapse}

The analytical function derived above assumes that the voltage escape associated with the synaptic current at the synaptic site is negligible. Because some voltage escape will inevitably be associated with somatic voltage clamp of dendritic synapses, it is therefore necessary to test how voltage escape affects the accuracy of the method. This was done using the equivalent cylinder model by progressively increasing the magnitude of the peak synaptic conductance at a given location. The results of such simulations are shown in Figure 6. As the synaptic conductance is increased, the voltage escape at the synapse progressively approaches the synaptic reversal potential, causing substantial distortions both in the current flowing at the synapse as well as in the current recorded at the soma. The charge recovery curves ob- tained from the same synapses show a progressive distortion and slowing after $t=0$. When comparing the decay time constant fit to the charge recovery curve with the actual time course of decay of the conductance (Fig. $6 F$ ), serious errors $(>10 \%)$ were found only for the largest conductances $(\geq 20 \mathrm{nS})$. These errors could be reduced further by changing the fit range; fits with a later onset produced greater accuracy (although, as pointed out above, this is not feasible for conductances that may contain a slow component). By contrast, the time constants fit to the decay of the current measured at the soma were seriously in error for all conductance values chosen; delaying the onset of the fit produced little improvement in accuracy.

These findings suggest that the voltage jump method can reliably extract the decay time course of the synaptic conductance over a wide range of magnitudes of the conductance, but that the substantial voltage escape associated with very large, highly localized synaptic conductances may reduce its accuracy. The amplitude of the voltage escape will depend not only on the magnitude of the conductance but also on the geometry of the cell as well as its electrical properties. To test the method rigorously, it is therefore of great importance to carry out simulations in compartmental models of real neurons, with realistic values for the membrane parameters and the synaptic conductance.

\section{Application to pyramidal cell geometries CA3 pyramidal cell}

Figure 7 shows a test of the voltage jump method in a detailed compartmental model of a CA3 pyramidal cell (Major et al., 1994). As shown previously (Major et al., 1994), a synaptic input placed on the distal apical dendrites is substantially filtered and attenuated by space-clamp errors (Fig. $7 B$ ). The voltage jump protocol was performed at a holding potential of $-65 \mathrm{mV}$. Because the analytical function assumes that the system is passive, it should not matter from which holding potential the jumps are made or which voltage is jumped to, as long as there is a change in synaptic driving force; the charge recovery curve is simply shifted downward on the $y$-axis by the difference in synaptic charge at the two holding potentials. By fitting the charge recovery with Equation 10, it was possible to extract the decay of the synaptic conductance with high accuracy $(<5 \%$ error; for details, see legend to Fig. 7). To determine the effect of high membrane conductance on the accuracy of the method, $R_{\mathrm{m}}$ was decreased from 180,000 to $20,000 \Omega \mathrm{cm}^{2}$ (which reduced the input resistance from 305 to $43.4 \mathrm{M} \Omega$ ). Under these conditions, as might be expected to occur in vivo because of tonic synaptic bombardment, the method extracted the decay time course of the conductance to within $2 \%$ error (data not shown). The method also maintained high accuracy under conditions of high series resistance (20 $\mathrm{M} \Omega$; Fig. $7 E-G)$. Note that in these simulations, the time course of the

\footnotetext{
$\leftarrow$

Figure 8. Simulation of a distributed inhibitory conductance in a CA3 pyramidal cell. A unitary connection made by a presynaptic "bitufted" inhibitory neuron is modeled, based on the work of Miles et al. (1996, their Fig. 2). The locations of the 8 individual contacts on apical and basal dendritic shafts are shown using dots in $A$. Each synaptic contact had an identical synaptic conductance, with a peak conductance of 1 nS and a reversal potential of 0 $\mathrm{mV}$. The rising time constant was $0.2 \mathrm{msec}$ in all cases, and the decay time constant was either a single exponential of $5 \mathrm{msec}(B-D)$ or a double exponential of $5 \mathrm{msec}(80 \%)$ and $30 \mathrm{msec}(20 \%) . B$ and $E$ compare the somatic clamp current with the perfectly clamped EPSC. The $20-80 \%$ rise times of the currents were $1.66 \mathrm{msec}$ in $B$ and $1.89 \mathrm{msec}$ in $E$. The decay of the somatic clamp current could be fit by a single exponential with $\tau=9.5$ and $22.2 \mathrm{msec}$, respectively. $C, F$, Recovered currents from successive voltage jumps from $-65 \mathrm{mV} . D$, $G$, Charge recovery curves, which have been fit with the analytical function. For the monoexponentially decaying conductance, the best fit of the analytical function was with the following parameters: $\tau_{\mathrm{v}_{1}}$ $=3.24 \mathrm{msec}(57 \%) ; \tau_{\mathrm{v}_{2}}=10.93 \mathrm{msec}(43 \%) ; \tau_{\mathrm{rise}}=0.66 \mathrm{msec}$; and $\tau_{\mathrm{dec}}=5.22 \mathrm{msec}$; fitting the decay of the charge recovery curve with a single exponential gave $\tau_{\mathrm{dec}}=5.16$ msec. For the conductance with a biexponential decay the best fit was with the following parameters: $\tau_{\mathrm{v}_{1}}=3.26 \mathrm{msec}(59 \%)$; $\tau_{\mathrm{v}_{2}}=11.11 \mathrm{msec}(41 \%) ; \tau_{\mathrm{rise}}=0.48 \mathrm{msec} ; \tau_{\mathrm{dec}_{1}}=5.17 \mathrm{msec}(77 \%)$; and $\tau_{\mathrm{dec}_{2}}=30.54 \mathrm{msec}(23 \%)$. A double-exponential fit to the decay of the charge recovery gave $\tau_{\text {dec }_{1}}=5.02 \mathrm{msec}(66 \%)$; and $\tau_{\mathrm{dec}_{2}}=30.48 \mathrm{msec}(34 \%)$.
} 


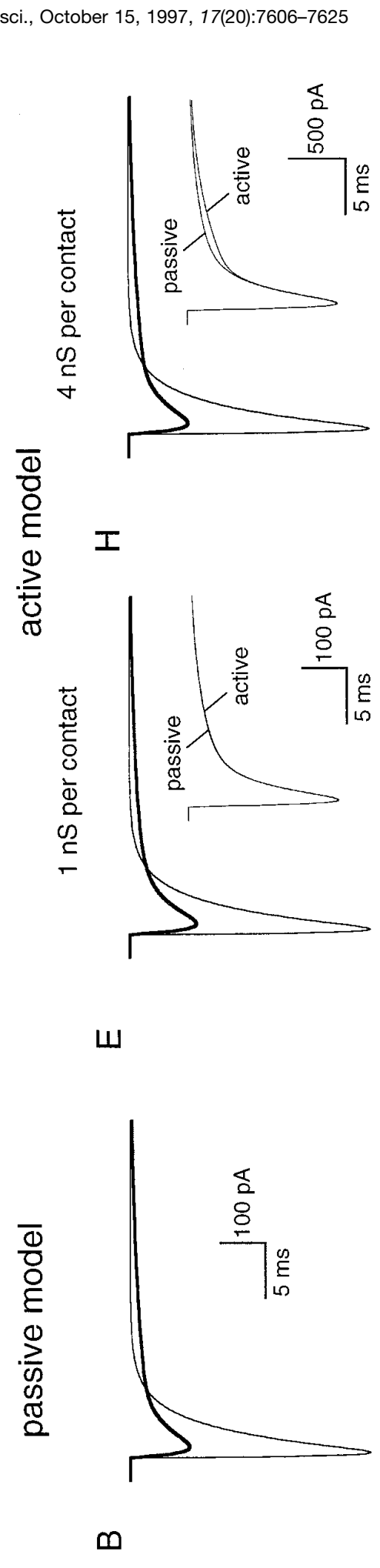

$\infty$

ᄂ
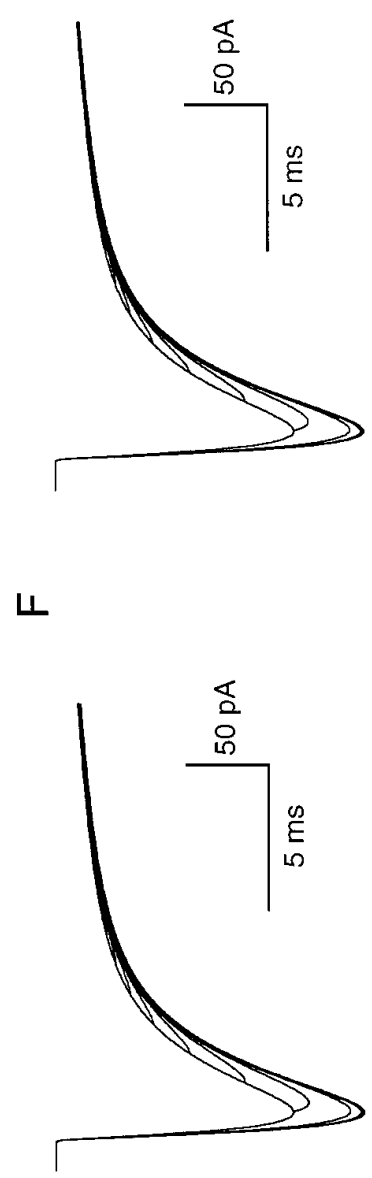

0

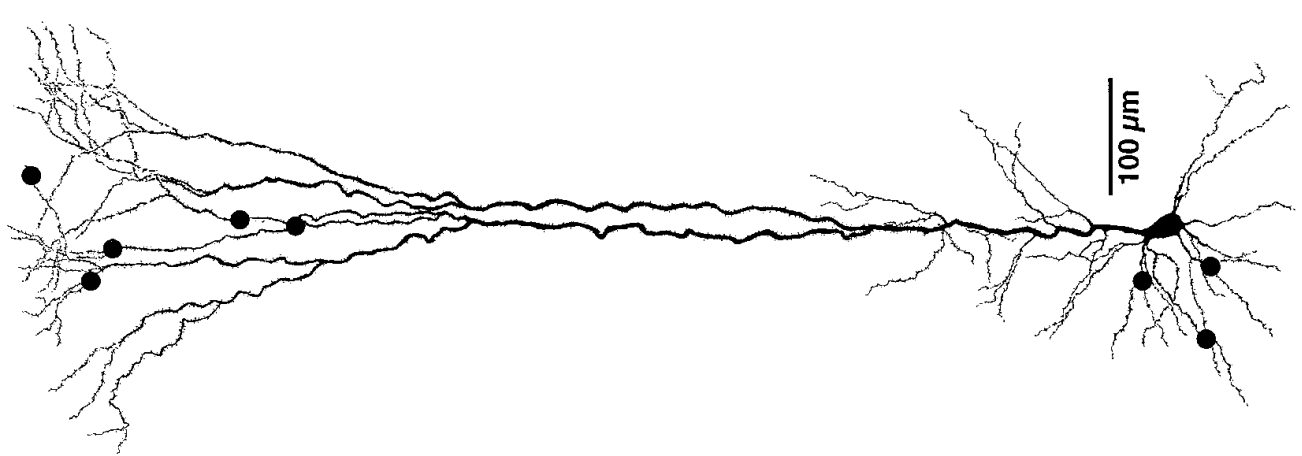

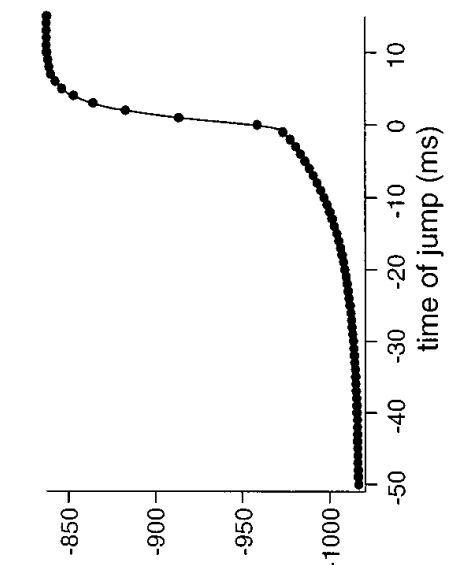

(1)

(อł) әблечо рәләлооәл

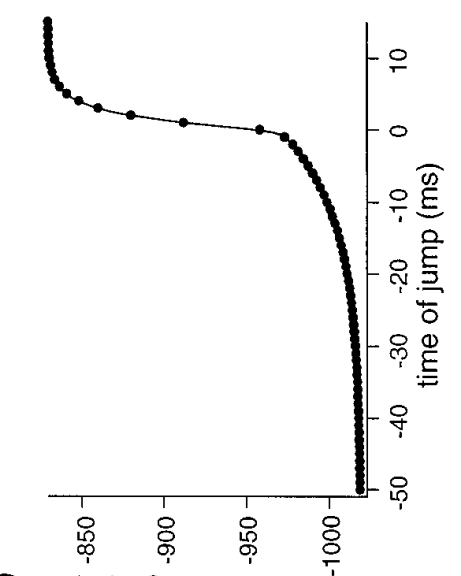

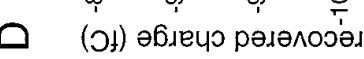

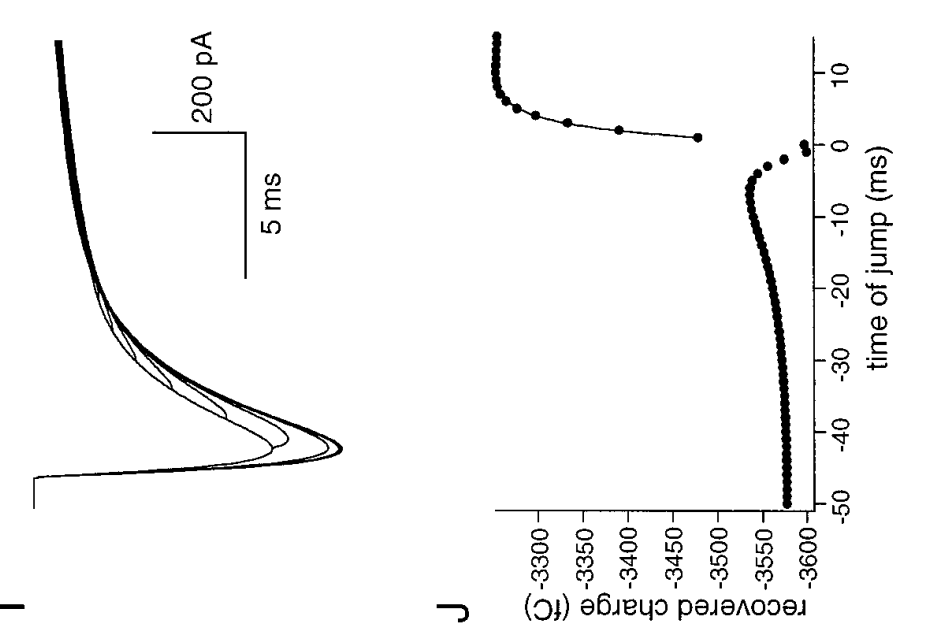

$\varangle$ 
initial phase of the charge recovery (and the $\tau_{\mathrm{v}}$ values extracted by fitting the analytical function) were much slower than with low series resistance, consistent with the greater effective electrotonic distance of the synapse in the high series resistance condition.

To test whether it is also possible to extract accurately the rise time of a slow synaptic conductance, an NMDA receptormediated EPSC (Kirson and Yaari, 1996) was simulated at the same synaptic location in Figure $7 H-J$. Although the decay of this synaptic current was not significantly distorted because of its slow time course, the rise time was slowed substantially (from $\tau_{\text {rise }}=$ 5.0 to $9.6 \mathrm{msec}$ ). The analytical function was able to extract the rise time (as well as the decay) to within $3 \%$ of its original value, indicating that the method may also be useful for this purpose.

To examine the effectiveness of the method for distributed conductances in the CA3 pyramidal cell, an inhibitory connection was simulated (Fig. 8), with the location of the contacts based on a reconstructed connection between an interneuron and a simultaneously recorded CA3 pyramidal cell (Miles et al., 1996, their Fig. 2). Either single- or double-exponentially decaying conductances were simulated at each contact (Pearce, 1993). When the decay of the synaptic conductance was double-exponential, the fast component of the decay was filtered more heavily than the slow component, such that the synaptic current measured at the soma could be fit with a single exponential with a $\tau$ intermediate to the two time constants of the conductance decay. Because the synapses in this simulation were at widely distributed electrotonic locations, when applying a somatic voltage jump each synapse experienced voltage transients with a different time course. This caused slight distortions of the rise time extracted with the analytical function. The decay appeared to be relatively little affected by this nonuniformity, as with both the single- and double-exponentially decaying conductances, it was possible to extract the time constants and their relative amplitudes to a high degree of accuracy ( $<5 \%$ error). To test the effect of the synaptic conductance kinetics on the accuracy of the method, we also performed simulations under the same conditions with a conductance decay time constant of $1 \mathrm{msec}$. The decay time constant extracted by the method was $1.01 \mathrm{msec}$ (data not shown), confirming that high accuracy could be maintained even with rapid input kinetics.

\section{Neocortical pyramidal cell}

The most stringent tests of the method were performed using a detailed compartmental model of a layer 5 pyramidal cell (Markram et al., 1997), a cell type that has one of the most extensive dendritic trees of any neuron in the brain. A morphologically reconstructed unitary input made by an adjacent, simultaneously recorded layer 5 pyramidal cell was simulated (Markram et al., 1997), which made eight contacts at widely dispersed electrotonic locations (mean $X=0.71$; range, 0.0631.4). When this distributed input was activated, the analytical function extracted the decay time constant of the synaptic conductance to within $5 \%$ error, despite substantial filtering of the synaptic current waveform (Fig. 9B-D). Errors remained small $(<5 \%)$ when the magnitude of the conductance at each contact was quadrupled to $4 \mathrm{nS}$, when the decay time constant of the synaptic conductance was reduced to $1 \mathrm{msec}$, and when the series resistance was increased to $5 \mathrm{M} \Omega$ (not shown).

To investigate the influence of active conductances on the method, the simulations were repeated incorporating an active membrane model of neocortical layer 5 pyramidal cells containing a variety of voltage-gated conductances, which reproduces the firing pattern of these neurons (Mainen and Sejnowski, 1996). Simulations with the active model at $1 \mathrm{nS}$ peak conductance per contact produced results that were very similar to those found with the passive model, consistent with the lack of distortion in the synaptic current (Fig. 9E, inset). When the peak synaptic conductance was increased to $4 \mathrm{nS} /$ contact, however, an obvious "boosting" component could be observed in the decay of the synaptic current (Fig. 9H, inset). The boosting current arose almost exclusively via activation of sodium and calcium conductances in the apical tuft branches (not shown); virtually no boosting was observed at the peak of the synaptic current, primarily because the measured peak is dominated by current from basal inputs, which are better clamped.

The extra charge contributed by the active conductances caused clear distortions in the charge recovery curve, with an extra component emerging in the onset of the charge recovery, representing jumps made just before the beginning of the synaptic conductance. The shape of this extra component results from a highly nonlinear process involving the increase in the driving force caused by the hyperpolarization, which is still weak enough at "late" times to permit activation of voltage-gated channels. Despite this distortion, the charge recovery after $t=0 \mathrm{msec}$ remained dominated by the decay of the synaptic conductance; when a single exponential was fit to this component, the decay was estimated to within $10 \%$. Similar results were obtained when the decay time constant was reduced to $1 \mathrm{msec}$ (not shown). In this model, therefore, the errors caused by active conductances de-

\footnotetext{
$\leftarrow$

Figure 9. Simulation of a distributed synaptic connection in an active layer 5 pyramidal cell model. A reconstructed synaptic connection made by a single presynaptic layer 5 pyramidal neuron is simulated, with 8 contacts (marked by dots in $A$ ) distributed on apical and basal dendritic spines (Markram et al., 1997). All synaptic conductances are identical $\left(\tau_{\text {rise }}=0.20 \mathrm{msec} ; \tau_{\mathrm{dec}}=2 \mathrm{msec}\right)$. The model either was passive $(B-D)$ or contained active conductances $(E-J)$, as described in Materials and Methods. The peak synaptic conductance at each contact was either 1 or 4 nS; the kinetics of the currents and charge recoveries obtained from the 1 and $4 \mathrm{nS}$ passive simulations was nearly identical, and therefore only the results from the $1 \mathrm{nS}$ simulation are shown. $B, E$, and $H$ compare the somatic clamp current at a holding potential of $-65 \mathrm{mV}$ with the perfectly clamped EPSC for the passive and active model. Insets in $E$ and $H$ compare the clamp current in the active model with that of the corresponding simulation in the passive model (same period as in the main panels; scale bars apply to the larger traces). Note that in the simulations with $1 \mathrm{nS}$ peak conductance, the active and passive models produce a virtually identical EPSC, whereas in the $4 \mathrm{nS}$ simulation the EPSC in the active model clearly shows an additional current component in the tail of the EPSC. The $20-80 \%$ rise times of the somatic EPSCs were $0.36 \mathrm{msec}$ in each case. The decay of the somatic EPSCs could be fit by a single exponential with $\tau=3.3 \mathrm{msec}$ in the passive simulations as well as in the active $1 \mathrm{nS}$ simulation, and with $\tau=3.7 \mathrm{msec}$ in the $4 \mathrm{nS}$ active simulation. $C, F, I$, Recovered currents from successive $-20 \mathrm{mV}$ hyperpolarizing voltage jumps from a holding potential of $-65 \mathrm{mV}$. $D$, $G, J$, Respective charge recovery curves measured from the recovered currents. In $D$ and $G$, the curves have been fit with the analytical function. The best fit in the passive model gave $\tau_{\mathrm{v}_{1}}=0.36 \mathrm{msec}(69 \%) ; \tau_{\mathrm{v}_{2}}=11.3 \mathrm{msec}(31 \%) ; \tau_{\text {rise }}=0.22 \mathrm{msec}$; and $\tau_{\text {dec }}=2.02 \mathrm{msec}$; whereas in the active model the values were $\tau_{\mathrm{v}_{1}}=0.23 \mathrm{msec}$ $(69 \%) ; \tau_{\mathrm{v}_{2}}=11.6 \mathrm{msec}(31 \%) ; \tau_{\text {rise }}=0.34 \mathrm{msec}$; and $\tau_{\mathrm{dec}}=1.90 \mathrm{msec}$. A single-exponential fit to the decay of the charge recovery gave $\tau_{\mathrm{dec}}=2.00 \mathrm{msec}$ in both cases. Because of the distortion of the charge recovery in the $4 \mathrm{nS}$ active simulation, a fit of the analytical function was not possible. However, the decay phase of the charge recovery was fit with a single exponential of $1.90 \mathrm{msec}$.
} 
A
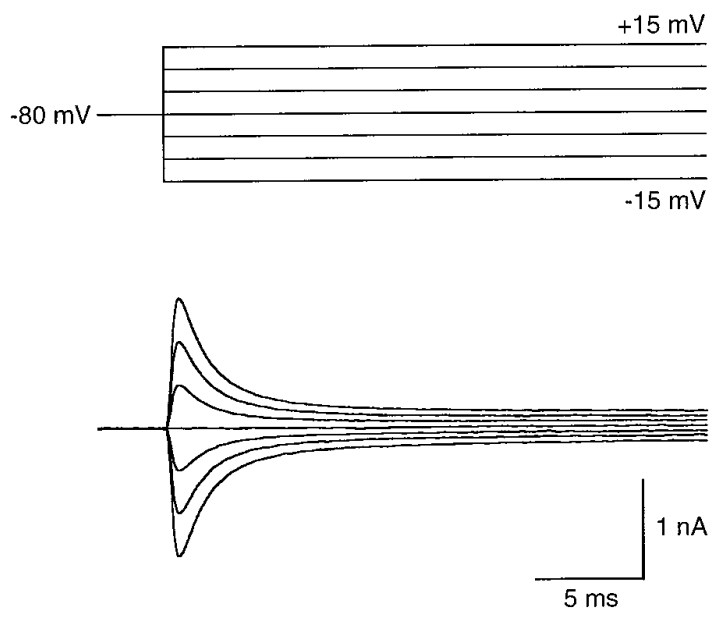

B

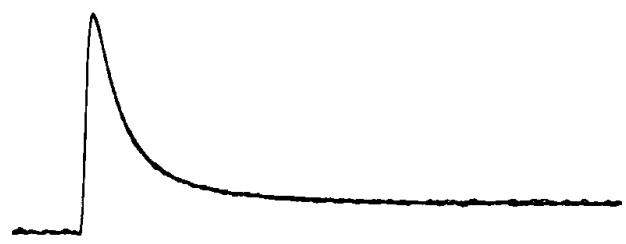

C
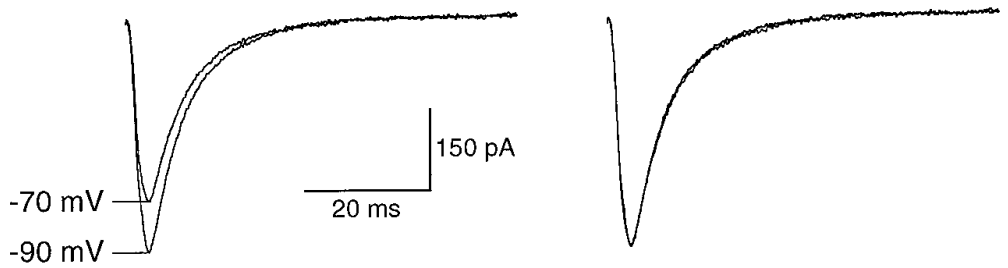

E

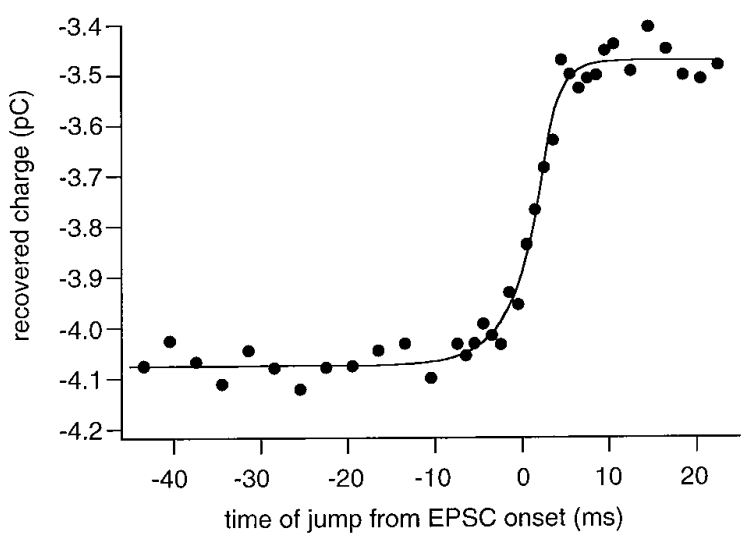

Figure 10. Determining the time course of excitatory synaptic conductances in neocortical pyramidal neurons using the voltage jump method. All traces taken from a somatic whole-cell recording of a layer 5 neocortical pyramidal neuron at $35^{\circ} \mathrm{C}$; the internal solution contained $1 \mathrm{~mm}$ QX-314 and $0.5 \mathrm{~mm}$ ZD 7288. $A$, The neuron was held at $-80 \mathrm{mV}$, and a series of voltage jumps (from -95 to $-65 \mathrm{mV}$ in $5 \mathrm{mV}$ steps) was given to test for membrane linearity, bracketing the voltage range used for determining the charge recovery. The resulting currents are shown below the voltage commands (average of 5 traces each; the series resistance of $6.0 \mathrm{M} \Omega$ was compensated by $90 \%$ ). $B$, The currents were scaled by the command voltage and superimposed to demonstrate linearity. An EPSC was evoked by stimulation of afferent fibers near the apical dendrite and is shown at two different holding potentials in $C$ (averages of 25 traces). $D$, The traces in $C$ have been scaled by their peak amplitudes and superimposed. The $20-80 \%$ rise times of the currents were 1.15 and $1.13 \mathrm{msec}$ at -70 and $-90 \mathrm{mV}$, respectively, and the decay time constants were 6.2 and $6.1 \mathrm{msec}$, respectively. $E$, Charge recovery curve obtained for this EPSC with jumps from -70 to $-90 \mathrm{mV}$. Each point represents the average of 21-26 separate trials. The values of the best fit using the analytical function (thick line) were $\tau_{\mathrm{v}}=3.36 \mathrm{msec} ; \tau_{\text {rise }}=0.54 \mathrm{msec}$; and $\tau_{\text {dec }}=1.47 \mathrm{msec}$. Fitting the decay of the charge recovery with a single exponential function gave $\tau_{\mathrm{dec}}=1.59 \mathrm{msec}$.

pend on various factors, particularly the size of the synaptic conductance (Fig. 9E,H) and the holding potential. These findings demonstrate that care must be taken to choose the appropriate voltage range over which to carry out the voltage jumps, and that tests must be done to evaluate the possible contribution of voltage-gated conductances.

\section{Experimental application of the voltage jump method to neocortical pyramidal cells}

The voltage jump method was used to determine experimentally the time course of excitatory synaptic conductances in layer 5 neocortical pyramidal cells. We evoked EPSCs resulting from the

Figure 11. Attenuation of synaptic currents and estimation of synaptic charge in layer 5 pyramidal cells. The location of the 5 synapses used in the simulation is shown by arrowheads in $A$. All synapses had identical conductances (peak $\mathrm{g}_{\text {syn }}=1.0 \mathrm{nS} ; \tau_{\text {rise }}=0.20 \mathrm{msec}$; and $\tau_{\text {dec }}=2 \mathrm{msec}$ ), and each synapse was activated individually. The somatic holding potential was at the resting potential $(-70 \mathrm{mV})$. $B$, Voltage escape at the synapse $\left(V_{\text {syn }}\right)$. $C$, Synaptic current flowing at the synapse $\left(I_{\text {syn }}\right)$. Note that the reduction in driving force as a consequence of the voltage escape causes a corresponding reduction in the amplitude of the synaptic current. $D$, Synaptic current measured at the soma $\left(I_{\text {soma }}\right)$ after activation of synapses at different locations. Note the striking distortion and reduction in peak amplitude of the currents originating at progressively more distal locations. The $20-80 \%$ rise times of the synaptic currents measured at the soma were soma synapse, $0.18 \mathrm{msec} ; 30 \mu \mathrm{m}, 0.25 \mathrm{msec} ; 100 \mu \mathrm{m}, 0.37 \mathrm{msec} ; 300 \mu \mathrm{m}, 0.78 \mathrm{msec}$; and $1000 \mu \mathrm{m}$, $3.12 \mathrm{msec}$. The decay time constants for somatic currents originating at the different locations were soma synapse, $2.00 \mathrm{msec} ; 30 \mu \mathrm{m}, 2.24 \mathrm{msec} ; 100 \mu \mathrm{m}$, $2.62 \mathrm{msec} ; 300 \mu \mathrm{m}, 4.14 \mathrm{msec}$; and $1000 \mu \mathrm{m}, 12.6 \mathrm{msec}$. E. Attenuation of voltage in response to a somatic voltage step at different locations in the dendritic tree. $F$, Shift of apparent synaptic reversal potential with increasing distance of the synapse from the somatic recording site (reversal potential of the synapse set to $0 \mathrm{mV}$ ). $G$ compares the attenuation of synaptic charge predicted from reversal potential shifts with the actual attenuation of synaptic charge. The predicted attenuation factor $(\alpha)$ was calculated according to Equation 11, and the actual attenuation factor was determined by dividing the integral of current recorded at the soma by the integral of the current flowing at the synapse. Note that for synapses at all distances the predicted and actual values fall along the unity line. $H$ compares the predicted charge with charge associated with the perfectly clamped EPSC. Each point represents the predicted charge divided by the synaptic charge expected with perfect clamp for synapses at different locations, and for three different peak amplitudes of the synaptic conductance $(0.1,1.0$, and $4.0 \mathrm{nS})$. 


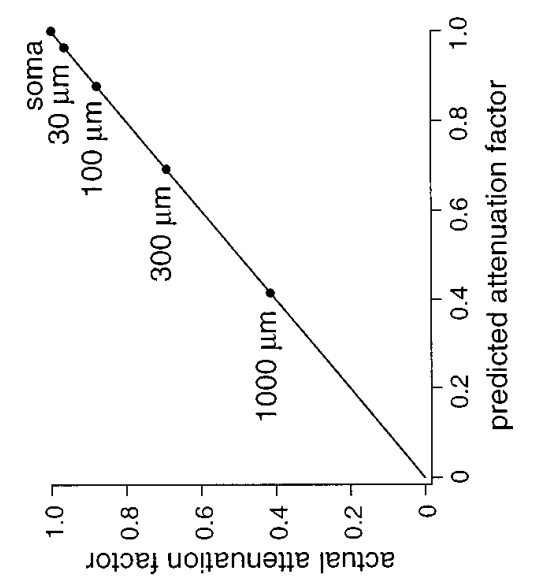

(

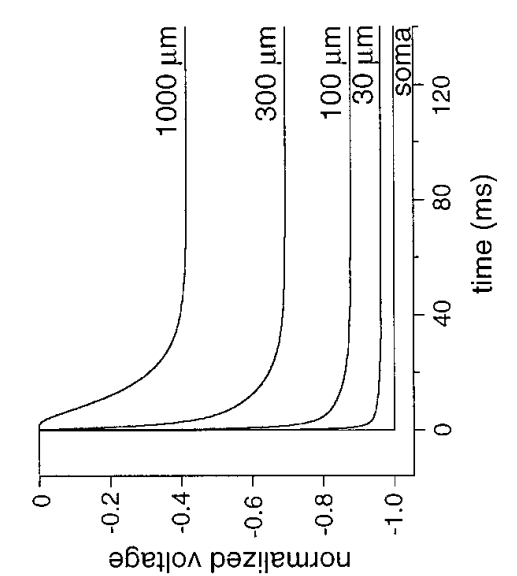

ш

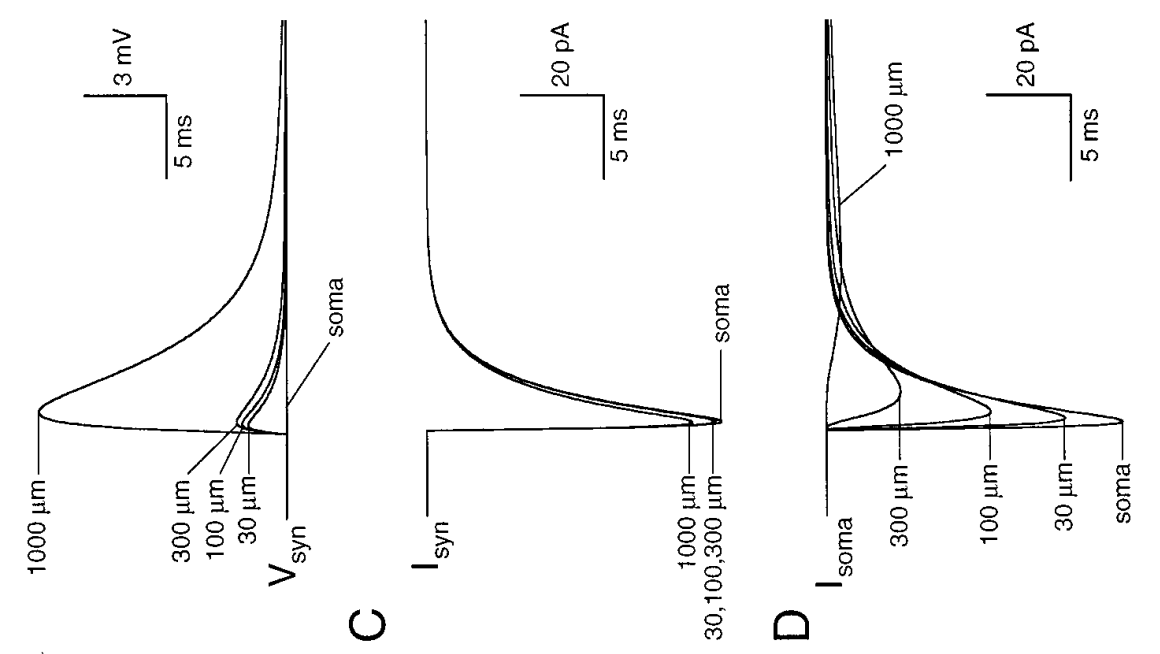

$\infty$

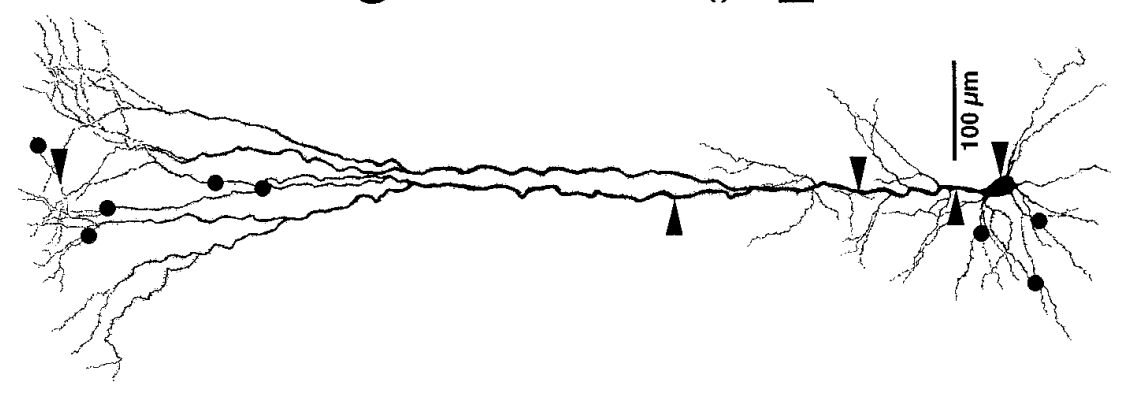

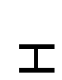

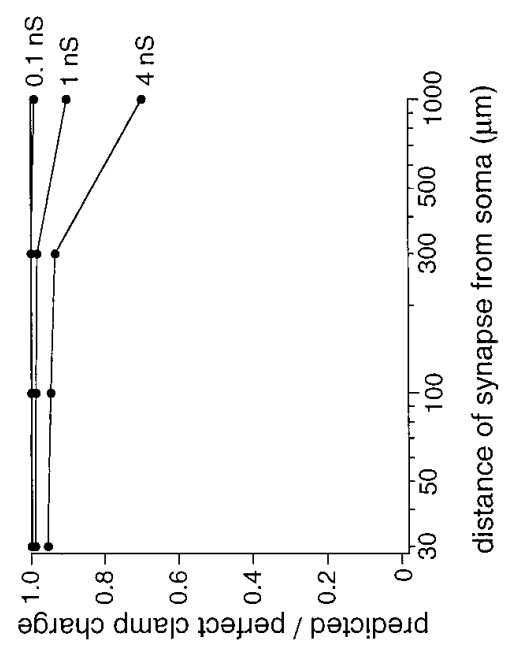

工

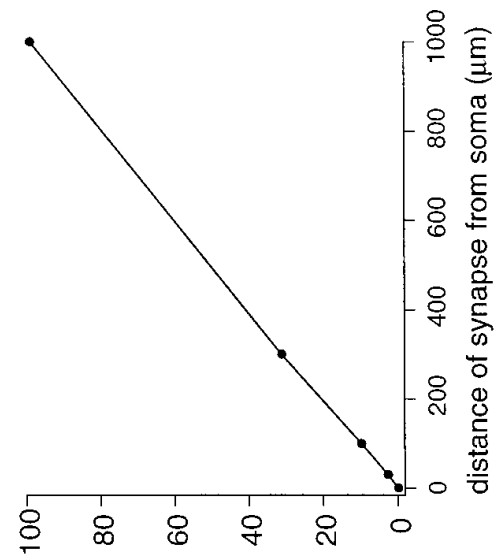

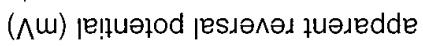

レ 
activation of only one or a few presynaptic fibers (peak amplitude, $546 \pm 50 \mathrm{pA}$ at $-70 \mathrm{mV} ; n=25)$. The evoked EPSCs had an average $20-80 \%$ rise time of $0.89 \pm 0.03$ (range, $0.55-1.20$ ) $\mathrm{msec}$ at $-70 \mathrm{mV}$, and their decay could be fit well using a single exponential function with a time constant of $3.83 \pm 0.24$ (range, 2.1-6.3) msec. The linearity of the membrane between -70 and $-90 \mathrm{mV}$ was examined by recording the membrane currents in response to a series of depolarizing and hyperpolarizing voltage jumps of different amplitudes starting from a holding potential of $-80 \mathrm{mV}$. When scaled by the jump amplitude, these currents superimposed well for jumps of different amplitude (see Fig. $10 B)$. To check for distortions in the EPSC caused by activation of voltage-gated conductances, the time course of the EPSC was compared at -70 and $-90 \mathrm{mV}$. At $-90 \mathrm{mV}$, the $20-80 \%$ rise time was $0.86 \pm 0.04 \mathrm{msec}(p=0.07$, paired $t$ test $)$, and the decay time constant was $3.74 \pm 0.22 \mathrm{msec}(p=0.06$; see Fig. $10 C, D)$. To confirm that activation of voltage-gated conductances did not affect the synaptic current and to assess possible distortions caused by voltage escape, the synaptic conductance was reduced by application of a submaximal concentration $(40 \mu \mathrm{M})$ of the noncompetitive AMPA receptor antagonist GYKI 52466 (Paternain et al., 1995). While the peak amplitude of the EPSC at -70 $\mathrm{mV}$ was reduced to $24 \pm 3 \%(n=3)$ compared with control, the $20-80 \%$ rise time and decay time constant of the EPSC were $106 \pm 2 \%(p=0.08)$ and $108 \pm 9 \%(p=0.4)$ of the control values, respectively. These findings indicate that the EPSCs were not substantially distorted by voltage escape or by the activation of voltage-gated conductances.

The voltage jump protocol was applied using jumps between -70 and $-90 \mathrm{mV}$. Jumps at different times relative to synaptic stimulation were interleaved, and a large number of individual sweeps were averaged for each jump time to reduce the contribution of noise associated with synaptic variability. The resulting charge recovery curves were fit with Equation 10, as shown in Figure $10 E$. Single-exponential functions provided a good fit to both the onset and offset of the curve, and it was usually necessary to constrain $\tau_{\text {rise }}$ to $0.1-0.6 \mathrm{msec}$. The time constant of the voltage at the synapse was $2.93 \pm 0.44 \mathrm{msec}$, and the decay time constant of the synaptic conductance was $1.74 \pm 0.18 \mathrm{msec}(n=8)$. The SEM predicted by Monte Carlo error analysis (see Materials and Methods) was $0.24 \mathrm{msec}$ for $\tau_{\mathrm{v}}$ and $0.28 \mathrm{msec}$ for $\tau_{\mathrm{dec}}$.

\section{Estimating the attenuation of synaptic charge}

Although the voltage jump method provides the kinetics of the synaptic conductance under conditions of inadequate space clamp, it offers no direct information about its magnitude. The peak amplitude of the conductance can be calculated, however, if the total synaptic charge is known in addition to the conductance kinetics. Determining the total synaptic charge from the somatically recorded current is possible given the attenuation of synaptic charge, $\alpha$ (introduced in Eqs. 1 and 5). Analytical solutions demonstrate that in a linear system the attenuation of synaptic charge from the synapse to the soma is equivalent to the attenuation of steady-state voltage from the soma to the synapse (Redman, 1973; Rinzel and Rall, 1974; Carnevale and Johnston, 1982; Jack et al., 1983; Rall and Segev, 1985; Major et al., 1993). If the reversal potential of the synaptic conductance is known, it is possible in principle to estimate the attenuation of steady-state voltage between the soma and the synapse in any geometry by comparing the apparent synaptic reversal potential measured at the soma with the expected value (Carnevale and Johnston, 1982; Jack et al., 1983; Rall and Segev, 1985). Here we estimate the attenuation factor $\alpha$ using the layer 5 pyramidal cell model and provide quantitative predictions of the magnitude of errors in $\alpha$ resulting from the voltage escape caused by having a finite synaptic conductance.

Figure 11 shows the attenuation of the synaptic current, the voltage escape at the synapse, and the resulting distortion of the current flowing at the synapse for five identical synapses at different locations in the layer 5 pyramidal cell model. Synaptic charge under perfect clamp will differ from the charge measured by somatic voltage clamp because of (1) the attenuation of synaptic charge between synapse and soma, $\alpha$, and (2) the reduction in synaptic driving force caused by voltage escape. The attenuation of voltage in the dendritic tree during a somatic voltage step at the five synaptic locations is shown in Figure $11 E$. This attenuation causes a corresponding shift in the apparent reversal potential of the synaptic current, shown in Figure $11 F$. The steady-state attenuation factor $\alpha$, representing the attenuation of voltage from soma to synapse, can then be calculated according to the following equation (cf. Carnevale and Johnston, 1982; Rall and Segev, 1985):

$$
\alpha=\frac{-V_{\text {rest }}+E_{\text {rev }}}{-V_{\text {rest }}+E_{\text {rev }}+\Delta E_{\text {rev }}},
$$

where $E_{\mathrm{rev}}$ is the reversal potential of the synaptic conductance, $\Delta E_{\text {rev }}$ is the shift in reversal potential from the expected value, and $V_{\text {rest }}$ is the resting potential of the cell. Confirmation that the value of $\alpha$ is identical to the attenuation of the charge associated with the synaptic current as it spreads from the synapse to the soma is provided in Figure $11 G$, where at each synaptic location the attenuation predicted from the reversal potential shift is identical to the actual attenuation of the charge flowing at the synapse, as expected from theory.

As pointed out above, the synaptic charge predicted from reversal potential shifts will not be identical to the charge expected under perfect voltage-clamp conditions because the voltage escape distorts the current flowing at the synapse by reducing its driving force (Fig. 11B,C). The magnitude of this error depends on the size of the synaptic conductance and the electrotonic location of the synapse. This is shown in Figure $11 \mathrm{H}$, which compares the synaptic charge predicted from Equation 11 with the synaptic charge expected under perfect voltage clamp for synapses at different distances and with different peak conductances. The predicted charge generally corresponds closely to the actual synaptic charge $(\leq 10 \%$ error for the most distal $1 \mathrm{nS}$ synapses), with the error converging toward zero as the conductance becomes smaller. In summary, these simulations describe a procedure that provides a relatively accurate measure of synaptic charge in any neuronal geometry, assuming that (1) the neuron behaves passively; (2) the reversal potential of the synaptic current is known; (3) the neuron is held at resting potential; and (4) the voltage escape associated with the synaptic current is relatively small. If these conditions hold, and the time course of the conductance is known (e.g., from using the voltage jump method), then it is possible to provide an estimate of the peak amplitude of the conductance.

\section{DISCUSSION}

We have demonstrated that measuring the recovery of synaptic charge with a series of voltage jumps can provide several important pieces of information: charge recovered by jumps made before the onset of the synaptic conductance reveals the voltage 
change at the synapse in response to the voltage step; and charge recovered by jumps made after the onset of the synaptic conductance reveals the kinetics of the conductance. We describe a simple analytical function which makes it possible to extract these features from experimental data, independent of the neuronal geometry. This approach therefore circumvents the serious distortions in the kinetics of the synaptic current caused by spaceclamp errors and provides an index of the electrotonic location of the synapse. We use the method to estimate the decay time course of the excitatory synaptic conductance in neocortical pyramidal cells, where space-clamp problems are severe for most synapses. We also show that by combining the method with an estimate of charge attenuation, it is possible in principle to reconstruct all aspects of the synaptic conductance waveform.

\section{Comparison with previous approaches}

Smith et al. (1967) used phase changes in a carrier sine wave applied at the soma to detect membrane impedance changes during synaptic potentials in motoneurons. Although this technique resolved the time course of conductance changes at proximal synapses, it was incapable of detecting distal conductance changes (as predicted theoretically by Rall, 1967). This is because the frequency of the carrier signal must be high to achieve sufficient time resolution, and consequently it rapidly attenuates as it spreads into the dendrites. In contrast, the present method uses a voltage transient with predominantly low-frequency components (the voltage step response) as a "windowing function," which is shifted in small steps over the synaptic conductance, conserving both high sensitivity to distal conductance changes and arbitrarily high time resolution. As a consequence of the need for multiple sweeps, the method cannot measure sweep-to-sweep fluctuations in conductance kinetics but instead reports the mean conductance time course of the active synapses.

Another approach is to estimate the filtering of synaptic currents using compartmental models of neurons (Johnston and Brown, 1983; Hestrin et al., 1990; Jonas et al., 1993; Spruston et al., 1993; Soltesz et al., 1995; Mainen et al., 1996). Given such estimates, it is possible in principle to determine the synaptic conductance time course by "working backward" from the measured current with a compartmental model of the same neuron. However, even the most carefully conditioned models still suffer from potentially serious nonuniqueness in the model parameters (Major et al., 1994). Furthermore, the location of the active synapses is usually unknown and difficult to determine. Consequently the range of error estimates is relatively broad, even for synaptic connections at which good estimates exist for the location of active synapses (Jonas et al., 1993). By contrast, the present method is independent of neuronal geometry and thus requires no knowledge of the electrotonic structure of the neuron being recorded from (as long as care is taken to exclude major sources of error). However, combining the voltage jump approach with compartmental modeling may be very powerful, as discussed below.

Several groups have used the response to single voltage jumps at the soma, either alone (Llano et al., 1991) or interacting with synaptic conductances (Hestrin et al., 1990; Isaacson and Walmsley, 1995; Sah and Bekkers, 1996) to estimate the filtering of synaptic currents (also see Silver et al., 1995). Llano et al. (1991) proposed that decay time constants of synaptic currents that were slower than the characteristic charging time constant of the distal compartment of juvenile Purkinje cells are not distorted by spaceclamp problems. Their two-compartment model is not, however, useful for synaptic conductances with decay kinetics comparable to or faster than the charging time constant and is unlikely to be applicable to other neuronal geometries. Somatic voltage jumps have also been used either to eliminate synaptic driving force (Isaacson and Walmsley, 1995; Sah and Bekkers, 1996) or to activate $\mathrm{Mg}^{2+}$ block of NMDA receptors (Hestrin et al., 1990). The rate of the resulting relaxation in the somatic synaptic current ("switch-off") provides a measure of the electrotonic location of the synapse. However, to make quantitative predictions about the filtering of the synaptic current based on the switch-off, a compartmental model of the cell is required (Sah and Bekkers, 1996).

Finally, dendritic recording of synaptic currents (Häusser, 1994) can be used to reduce the electrotonic distance between the clamp site and dendritic synapses. However, because only synapses close to the dendritic recording site will be well clamped, it is necessary to selectively activate nearby synapses or to select spontaneous events based on electrotonic proximity (Häusser, 1994). In principle one could combine dendritic voltage-clamp recording with the voltage jump method to improve resolution of the most distal synaptic conductances.

\section{Sources of error}

The voltage jump method assumes that the synaptic conductance is identical from one jump to the next. Real synapses, however, display trial-to-trial variability in amplitude and time course. This variability introduces noise into the charge recovery curve. The influence of synaptic and instrumental noise on the accuracy of the parameter estimates will be different for each experimental situation. Certain synaptic connections are more favorable than others with respect to synaptic variability; connections that make many contacts with high release probability (such as the cerebellar climbing fiber synapse) will be particularly suited to the method, owing to the resulting low synaptic coefficient of variation. When increasing the number of active synapses in an attempt to reduce variability in the synaptic response, a tradeoff is expected between noise in the charge recovery and problems associated with voltage escape; the larger the synaptic signal, the better the resolution of the method, but also the greater the risk that voltage escape may distort the synaptic current (see below). If noise is a problem, then collecting more sweeps or increasing voltage jump amplitude is usually preferable whenever possible. The cortical synapses studied here show considerable trial-to-trial variability (Markram et al., 1997); therefore, averages of many individual sweeps were necessary to construct charge recovery curves with acceptable noise levels.

When fitting charge recovery curves contaminated by noise, several issues must be considered. First, deciding the number of exponential components required may be a problem, because separation of closely spaced exponentials can be difficult even if data are free of noise (Provencher, 1976). Changing the number of exponential components for one part of the curve can affect the relative amplitudes of other exponential components (cf. Eq. 10). Also, by assuming a monoexponential decay of the synaptic conductance when it is in fact biexponential, the "effective" single $\tau_{\mathrm{dec}}$ (see Major, 1993) may be overestimated. Second, estimates of $\tau_{\text {rise }}$ may be associated with considerable uncertainty, especially if it is fast, because information about the rise time is contained in only a few points of the charge recovery curve. If the rise time is an important unknown parameter, then greater time resolution is needed around $t=0 \mathrm{msec}$ (i.e., more closely spaced jumps).

Even under the noise-free conditions of the simulations, the 
voltage jump method does not extract the time course of the synaptic conductance with perfect accuracy. The reason for this discrepancy is that the method measures the kinetics not of the synaptic current expected under perfect voltage-clamp conditions but, rather, of the actual current flowing at the synapse, which will be distorted by voltage escape. The extent of voltage escape will therefore determine how well the kinetic parameters extracted by the method reflect those of the actual synaptic conductance. In simulations using models of neurons with realistic synaptic conductances, errors caused by voltage escape were relatively small $(<10 \%)$. Nevertheless, voltage escape may represent a greater problem under certain conditions, for example, when activating a large number of closely spaced synapses. This can be assessed by applying a nonsaturating dose of a noncompetitive antagonist (or a competitive antagonist with slow dissociation kinetics) to reduce the size of the synaptic conductance. If the shape of the measured synaptic current does not differ after this treatment, then the effects of voltage escape can be safely neglected.

Active membrane conductances may also distort the charge recovery. The contribution of active conductances will depend primarily on their $I-V$ relation. Also, if their activation kinetics are slow relative to the synaptic conductance kinetics, or if the channels are located far from the synapses (e.g., in the axon), then their contribution will be less important. Interestingly, despite the distortions in the charge recovery observed in the neocortical pyramidal cell model, the synaptic conductance decay was relatively faithfully reported, indicating that it predominates under these conditions. Nevertheless, to fit Equation 10 reliably, both the jumps and the synaptic current should show passive behavior, as demonstrated in our experiments. This was ensured by recording at hyperpolarized potentials and by applying intracellular blockers via the recording pipette.

\section{Potential applications of the method}

The voltage jump method should be useful for determining the time course of synaptic conductances lacking appreciable voltage dependence in any neuron where space clamp is not guaranteed. The relative insensitivity of the method to membrane conductance and series resistance means that it could be used to measure the time course of synaptic conductances in vivo, where membrane conductance is higher (because of tonic synaptic activity) and where good space-clamp conditions are especially difficult to achieve. The method should also be useful for monitoring changes in synaptic conductance time course when space-clamp conditions are not constant, such as during development. Using the method, it should be possible to test whether distal synaptic currents have slower kinetics than proximal ones (Pearce, 1993), a mechanism that may compensate for electrotonic attenuation of distal inputs (Jack et al., 1981; Stricker et al., 1996). The method is not restricted to examining synaptic conductances; the kinetics of any conductance that lacks appreciable voltage dependence, such as certain sodium-activated (Koh et al., 1994) or calciumactivated (Sah and Bekkers, 1996) potassium conductances, can also be determined.

The ability of the method to estimate the time course of the voltage change at the conductance location in response to a somatic voltage step should be particularly useful, because it offers an index of the electrotonic distance of the conductance. This allows one to compare the relative electrotonic distance of different synapses (cf. Sah and Bekkers, 1996). Furthermore, because the time course of the voltage change at the synapse is known, one can estimate the physical distance of the synapses from the recording site with a compartmental model. Another possibility is to use arbitrary conductance changes to map the electrotonic structure of the dendritic tree. For example, focal application of neurotransmitter (to generate a conductance) could be combined with voltage jumps to map the electrotonic geometry of the neuron in regions that may be inaccessible to direct recording, providing constraints for compartmental models of such neurons. Finally, the method could also be used with arbitrary voltage command waveforms (as long as the response can be described by sums of exponentials). This may allow prediction of the filtering experienced by physiologically relevant signals, such as action potentials and synaptic potentials, as they propagate in the dendritic tree.

\section{Rapid decay time course of the excitatory synaptic conductance in neocortical pyramidal cells}

The relatively rapid decay time course of the excitatory synaptic conductance in neocortical pyramidal cells we have estimated is consistent with recordings of selected spontaneous EPSCs (Stuart and Sakmann, 1995), assuming residual space-clamp error in the latter measurements, as well as with the deactivation kinetics of AMPA-type glutamate receptor channels in these neurons (Hestrin, 1993; Jonas et al., 1994), correcting for temperature using a $Q_{10}$ of $\sim 2$ (Silver et al., 1996). This result has important physiological implications. The decay of the EPSC largely determines the decay of the EPSP at its site of generation (Rall, 1967; Jack et al., 1983; Softky, 1994). The rapid decay of the conductance ensures that the time window for local synaptic integration in the dendritic tree remains brief, consistent with the proposed role of cortical pyramidal cells as coincidence detectors of synaptic input (Abeles, 1991; Softky, 1994; König et al., 1996).

\section{REFERENCES}

Abeles M (1991) Corticonics: neural circuits of the cerebral cortex. New York: Cambridge UP.

Barbour B, Keller BU, Llano I, Marty A (1994) Prolonged presence of glutamate during excitatory synaptic transmission to cerebellar Purkinje cells. Neuron 12:1331-1343.

Borst JGG, Lodder JC, Kits KS (1994) Large amplitude variability of GABAergic IPSCs in melanotropes from Xenopus laevis-evidence that quantal size differs between synapses. J Neurophysiol 71:639-655.

Carnevale NT, Johnston D (1982) Electrophysiological characterization of remote chemical synapses. J Neurophysiol 47:606-621.

Finkel AS, Redman SJ (1983) The synaptic current evoked in cat spinal motoneurones by impulses in single group Ia axons. J Physiol (Lond) 342:615-632.

Forsythe ID, Barnes-Davies M (1993) The binaural auditory pathwayexcitatory amino-acid receptors mediate dual timecourse excitatory postsynaptic currents in the rat medial nucleus of the trapezoid body. Proc R Soc Lond [Biol] 251:151-157.

Harris NC, Constanti A (1995) Mechanism of block by ZD 7288 of the hyperpolarization-activated inward rectifying current in guinea-pig substantia nigra neurons in vitro. J Neurophysiol 74:2366-2378.

Häusser M (1994) Kinetics of excitatory synaptic currents in Purkinje cells studied using dendritic patch-clamp recording. Soc Neurosci Abstr 20:891.

Hestrin S (1993) Different glutamate receptor channels mediate fast excitatory synaptic currents in inhibitory and excitatory cortical neurons. Neuron 11:1083-1091.

Hestrin S, Nicoll RA, Perkel DJ, Sah P (1990) Analysis of excitatory synaptic action in pyramidal cells using whole-cell recording from rat hippocampal slices. J Physiol (Lond) 422:203-225.

Hines M (1993) NEURON - a program for simulation of nerve equations. In: Neural systems: analysis and modeling (Eeckman FH, ed), pp 127-136. Boston: Kluwer.

Isaacson JS, Walmsley B (1995) Receptors underlying excitatory synaptic transmission in slices of the rat anteroventral cochlear nucleus. J Neurophysiol 73:964-973.

Jack JJB, Redman SJ, Wong K (1981) The components of synaptic 
potentials evoked in spinal motoneurones by impulses in single group Ia afferents. J Physiol (Lond) 321:65-96.

Jack JJB, Noble D, Tsien RW (1983) Electric current flow in excitable cells. Oxford: Clarendon.

Johnston D, Brown TH (1983) Interpretation of voltage-clamp measurements in hippocampal neurons. J Neurophysiol 50:464-486.

Jonas P, Major G, Sakmann B (1993) Quantal components of unitary EPSCs at the mossy fibre synapse on CA3 pyramidal cells of rat hippocampus. J Physiol (Lond) 472:615-663.

Jonas P, Racca C, Sakmann B, Seeburg PH, Monyer H (1994) Differences in $\mathrm{Ca}^{2+}$ permeability of AMPA-type glutamate receptor channels in neocortical neurons caused by differential GluR-B subunit expression. Neuron 12:1281-1289.

Kirson ED, Yaari Y (1996) Synaptic NMDA receptors in developing mouse hippocampal neurones: functional properties and sensitivity to ifenprodil. J Physiol (Lond) 497:437-455.

Koh D-S, Jonas P, Vogel W (1994) $\mathrm{Na}^{+}$-activated $\mathrm{K}^{+}$channels localized in the nodal region of myelinated axons of Xenopus. J Physiol (Lond) 479:183-197.

König P, Engel AK, Singer W (1996) Integrator or coincidence detector? The role of the cortical neuron revisited. Trends Neurosci 19:130-137.

Llano I, Marty A, Armstrong CM, Konnerth A (1991) Synaptic and agonist-induced currents of Purkinje cells in rat cerebellar slices. J Physiol (Lond) 434:183-213.

Mainen ZF, Sejnowski TJ (1996) Influence of dendritic structure on firing pattern in model neocortical neurons. Nature 382:363-366.

Mainen ZF, Carnevale NT, Zador AM, Claiborne BJ, Brown TH (1996) Electrotonic architecture of hippocampal CA1 pyramidal neurons based on three-dimensional reconstructions. J Neurophysiol 76:1904-1923.

Major G (1993) Solutions for transients in arbitrarily branching cables: III. Voltage clamp problems. Biophys J 65:469-491.

Major G, Evans JD, Jack JJB (1993) Solutions for transients in arbitrarily branching cables: II. Voltage clamp theory. Biophys J 65:450-468.

Major G, Larkman AU, Jonas P, Sakmann B, Jack JJB (1994) Detailed passive cable models of whole-cell recorded CA3 pyramidal neurons in rat hippocampal slices. J Neurosci 14:4613-4638.

Markram H, Lübke J, Frotscher M, Roth A, Sakmann B (1997) Physiology and anatomy of synaptic connections between thick tufted pyramidal neurones in the developing rat neocortex. J Physiol (Lond) 500:409-440.

Mennerick S, Zorumski CF (1995) Presynaptic influence on the time course of fast excitatory synaptic currents in cultured hippocampal cells. J Neurosci 15:3178-3192.

Miles R, Toth K, Gulyás A, Hájos N, Freund T (1996) Differences between dendritic and somatic inhibition in the hippocampus. Neuron 16:815-823.

Nelson PG, Pun RYK, Westbrook GL (1986) Synaptic excitation in cultures of mouse spinal cord neurones: receptor pharmacology and behaviour of synaptic currents. J Physiol (Lond) 372:169-190.

Paternain AV, Morales M, Lerma J (1995) Selective antagonism of AMPA receptors unmasks kainate receptor-mediated responses in hippocampal neurons. Neuron 14:185-189.

Pearce RA (1993) Physiological evidence for two distinct $\mathrm{GABA}_{\mathrm{A}}$ responses in rat hippocampus. Neuron 10:189-200.

Peters A, Kaiserman-Abramof IR (1970) The small pyramidal neuron of the rat cerebral cortex: the perikaryon, dendrites and spines. Am J Anat 127:321-356.

Press WH, Teukolsky SA, Vetterling WT, Flannery BP (1992) Numerical recipes in $\mathrm{C}$ : the art of scientific computing, $\mathrm{Ed} 2$. Cambridge: Cambridge UP.

Provencher SW (1976) A Fourier method for the analysis of exponential decay curves. Biophys J 16:27-41.
Rall W (1967) Distinguishing theoretical synaptic potentials computed for different soma-dendritic distributions of synaptic input. J Neurophysiol 30:1138-1168.

Rall W (1969) Time constants and electrotonic length of membrane cylinders and neurons. Biophys J 9:1483-1508.

Rall W, Segev I (1985) Space-clamp problems when voltage clamping branched neurons with intracellular microelectrodes. In: Voltage and patch clamping with microelectrodes (Smith Jr TG, Lecar H, Redman SJ, Gage P, eds), pp 191-215. Bethesda, MD: American Physiological Society.

Redman S (1973) The attenuation of passively propagating dendritic potentials in a motoneurone cable model. J Physiol (Lond) 234:637-664.

Rinzel J, Rall W (1974) Transient response in a dendritic neuron model for current injected at one branch. Biophys J 14:759-790.

Rossi DJ, Alford S, Mugnaini E, Slater NT (1995) Properties of transmission at a giant glutamatergic synapse in cerebellum: the mossy fiber-unipolar brush cell synapse. J Neurophysiol 74:24-42.

Sah P, Bekkers JM (1996) Apical dendritic location of slow afterhyperpolarization current in rat hippocampal pyramidal neurons: implications for the integration of long-term potentiation. $\mathrm{J}$ Neurosci 16:4537-4542.

Schneggenburger R, Konnerth A (1992) GABA-mediated synaptic transmission in neuroendocrine cells: a patch-clamp study in a pituitary slice preparation. Pflügers Arch 421:364-373.

Silver RA, Traynelis SF, Cull-Candy SG (1992) Rapid-time-course miniature and evoked excitatory currents at cerebellar synapses in situ. Nature 355:163-166.

Silver RA, Farrant M, Cull-Candy SG (1995) Filtering of the synaptic current estimated from the time course of NMDA channel opening. Soc Neurosci Abstr 21:584.

Silver RA, Colquhoun D, Cull-Candy SG, Edmonds B (1996) Deactivation and desensitization of non-NMDA receptors in patches and the time course of EPSCs in rat cerebellar granule cells. J Physiol (Lond) 493:167-173.

Smith TG, Wuerker RB, Frank K (1967) Membrane impedance changes during synaptic transmission in cat spinal motoneurons. J Neurophysiol 30:1072-1096.

Softky W (1994) Sub-millisecond coincidence detection in active dendritic trees. Neuroscience 58:13-41.

Soltesz I, Smetters DK, Mody I (1995) Tonic inhibition originates from synapses close to the soma. Neuron 14:1273-1283.

Spruston N, Jaffe DB, Williams SH, Johnston D (1993) Voltage- and space-clamp errors associated with the measurement of electrotonically remote synaptic events. J Neurophysiol 70:781-802.

Spruston N, Jaffe DB, Johnston D (1994) Dendritic attenuation of synaptic potentials and currents: the role of passive membrane properties. Trends Neurosci 17:161-166.

Strichartz GR (1973) The inhibition of sodium currents in myelinated nerve by quaternary derivatives of lidocaine. J Gen Physiol 62:37-57.

Stricker C, Field AC, Redman SJ (1996) Statistical analysis of amplitude fluctuations in EPSCs evoked in rat CA1 pyramidal neurons in vitro. J Physiol (Lond) 490:419-441.

Stuart G, Sakmann B (1995) Amplification of EPSPs by axosomatic sodium channels in neocortical pyramidal neurons. Neuron 15:1065-1076.

Stuart G, Dodt H-U, Sakmann B (1993) Patch-clamp recordings from the soma and dendrites of neurons in brain slices using infrared video microscopy. Pflügers Arch 423:511-518.

Zhang S, Trussell LO (1994) Voltage clamp analysis of excitatory synaptic transmission in the avian nucleus magnocellularis. J Physiol (Lond) 480:123-136. 